International Journal of Uncertainty, Fuzziness and Knowledge-Based Systems Vol. 18, No. 6 (2010) 721-758

(C) World Scientific Publishing Company

DOI: $10.1142 / \mathrm{S} 0218488510006775$

\title{
LEARNING AUTOMATA-BASED ALGORITHMS FOR FINDING MINIMUM WEAKLY CONNECTED DOMINATING SET IN STOCHASTIC GRAPHS
}

\author{
JAVAD AKBARI TORKESTANI \\ Department of Computer Engineering, Islamic Azad University, Arak, Iran \\ j-akbari@iau-arak.ac.ir \\ MOHAMMAD REZA MEYBODI \\ Department of Computer Engineering and IT, Amirkabir University, Tehran, Iran \\ mmeybodi@aut.ac.ir \\ Received 28 September 2008 \\ Revised 26 August 2010
}

\begin{abstract}
A weakly connected dominating set (WCDS) of graph $G$ is a subset of $G$ so that the vertex set of the given subset and all vertices with at least one endpoint in the subset induce a connected sub-graph of $G$. The minimum WCDS (MWCDS) problem is known to be NP-hard, and several approximation algorithms have been proposed for solving MWCDS in deterministic graphs. However, to the best of our knowledge no work has been done on finding the WCDS in stochastic graphs. In this paper, a definition of the MWCDS problem in a stochastic graph is first presented and then several learning automata-based algorithms are proposed for solving the stochastic MWCDS problem where the probability distribution function of the weight associated with the graph vertices is unknown. The proposed algorithms significantly reduce the number of samples needs to be taken from the vertices of the stochastic graph. It is shown that by a proper choice of the parameters of the proposed algorithms, the probability of finding the MWCDS is as close to unity as possible. Experimental results show the major superiority of the proposed algorithms over the standard sampling method in terms of the sampling rate.
\end{abstract}

Keywords: Dominating set; weakly connected dominating set; stochastic graph; learning automata; distributed learning automata.

\section{Introduction}

The dominating set (DS) problems are a class of optimization problems which are widely used in wireless ad hoc networks. ${ }^{1-14}$ The minimum connected dominating set (MCDS) of the network topology graph forms a virtual backbone by which the routing overhead can be significantly reduced, where the number of vertices responsible for the route discovery and data transmission can be reduced to the 
number of vertices in the MCDS. ${ }^{6,9,12-16}$ A WCDS of a graph $G$ is a dominating subset of $G$ so that the vertex set of the given subset and all the vertices with at least one endpoint in the subset induce a connected sub-graph of $G$. Finding the $\operatorname{WCDS}^{1,2,4-6,8,10}$ is a well-known approach for clustering the wireless ad hoc networks, ${ }^{17,18-25}$ and was first proposed by Chen and Listman in Ref. 4. In this clustering method, the dominator nodes and their closed neighbors assume the role of the cluster-heads and cluster members, respectively. The structure of the network graph can be simplified using WCDS and made more succinct for routing in ad hoc networks. ${ }^{9,11}$ Dunbar et al. ${ }^{26}$ studied weakly connected domination in graphs and showed that the problem of finding a minimum size WCDS in a given graph is NP-Hard.

Chen and Listman ${ }^{4}$ first proposed a distributed approximation algorithm for clustering the wireless ad hoc networks by finding a near optimal solution to the minimum size WCDS problem. The proposed algorithm is also inspired by Guha and Khuller's centralized approximation algorithm ${ }^{8}$ for finding small connected dominating sets (CDS). Guha and Khuller ${ }^{11}$ proposed two centralized greedy heuristic algorithms with bounded performance guarantees for connected dominating set formation. In the first algorithm, the connected dominating set is grown from one node outward, and in the second algorithm, a WCDS is constructed, and then the intermediate nodes are selected to create a CDS. Chen and Liestman also proposed a zonal algorithm ${ }^{1,5}$ in which the graph is divided into regions. A WCDS is constructed for each region, and adjustments are made along the borders of the regions to produce a WCDS for the whole graph. Their algorithm for the partitioning phase is partly based on the algorithm proposed by Gallager et al. ${ }^{7}$ for finding the minimum spanning tree in weighted graphs. Han and $\mathrm{Jia}^{2}$ also proposed an area-based distributed algorithm for WCDS construction in vertex-weighted graphs with constant approximation ratio, linear time and message complexity. While it has a lower message complexity than the zonal algorithm proposed by Chen and Listman, it outperforms the mentioned algorithm. Alzoubi et al. ${ }^{6}$ presented two distributed algorithms for finding a WCDS in ad hoc networks. The first algorithm was implemented by first electing a leader among the nodes, which was going to be the root of a spanning tree. The spanning tree is then traversed and the dominator nodes are selected. But the distributed leader election is extremely expensive in practice, and exhibits a very low degree of parallelism. The second algorithm first constructs a maximum independent set (MIS) by an iterative labeling strategy, and then modifies the formed MIS by selecting one intermediate node between each pair of dominators separated by exactly three hops.

In all existing methods proposed for solving the minimum WCDS problem, either the WCDS is constructed for unweighted graphs or it is assumed that the graph is deterministic and so the weight associated with the graph vertices is fixed and does not vary with time. Since the characteristics of the wireless ad hoc networks are stochastic, unpredictable and time-varying, ${ }^{27-29}$ such an assumption can 
not hold true in realistic applications. Therefore, the stochastic graphs (which are described later in Subsection 2.2) are more appropriate structures for modeling the realistic behaviors and dynamics of the ad hoc networks. The minimum WCDS problem in deterministic graphs has been widely studied in the literature and a host of solutions are available, ${ }^{1,2,4-6,8,10}$ but to the best of our knowledge, the idea of solving the minimum WCDS problem in a stochastic weighted graph is discussed for the first time here. In this paper, the stochastic minimum weakly connected dominating set problem is first introduced and then five learning automata-based approximation algorithms are proposed for solving the mentioned problem, when the probability distribution function (PDF) of the weight of the vertices is unknown. Each of the proposed algorithms is composed of the several stages, and the vertices that must be sampled at each stage are randomly selected by the learning automata. As the proposed algorithms proceed, the sampling process is concentrated on the vertices that construct the WCDS with the minimum expected weight. Hence, the proposed learning automata-based algorithms are able to reduce the number of samples needs to be taken from the graph by reducing the rate of unnecessary samples taken from the vertices which are not included in the MWCDS. To show the performance of the proposed algorithms, we have conducted two groups of experiments on stochastic graphs. In the first group, the proposed algorithms are tested on several stochastic benchmark graphs. The second group of experiments investigates the scalability of the proposed algorithms on a subset of dense stochastic graphs. In all experiments conducted in this paper, the number of samples taken by the proposed algorithms from the stochastic graphs is compared with that of the standard sampling method. Experimental results show the huge superiority of the proposed algorithms over the SSM in terms of the sampling rate.

The rest of the paper is organized as follows. In the next section, the dominating sets, stochastic graphs, and learning automata are briefly introduced. The proposed learning automata-based algorithms are described in Sec. 3. In Sec. 4, the convergence results of the first proposed algorithm are studied. In this section, strong convergence theorems in which the convergence of the first proposed algorithm to the optimal solution is proved are first presented. Then, the relationship between the learning rate and the convergence rate of algorithm is studied. The performance of the proposed algorithms is evaluated through the simulation experiments in Sec. 5. Section 6 concludes the paper.

\section{Preliminaries and Definitions}

In this section, to provide a sufficient background for the remainder of the paper, we present a brief overview of the dominating set problems and their applications in communication networks, stochastic graph, and learning automata and its variations. 


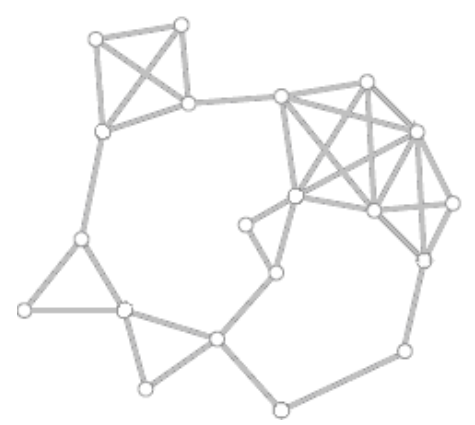

Fig. 1(A) A sample unit disk graph.

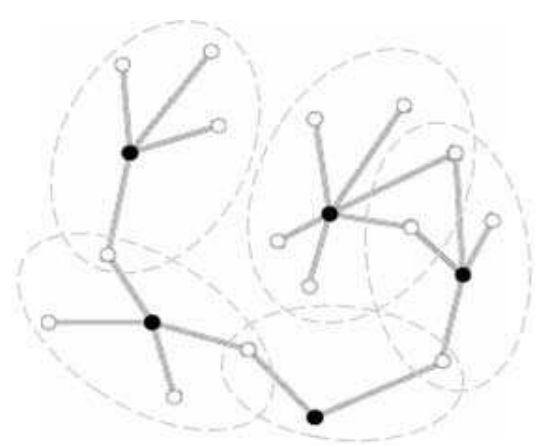

Fig. 1(B) The weakly connected dominating set.

\subsection{Dominating sets}

Dominating set $S$ of a given graph $G=(V, E)$ is a subset of vertex set $V$, such that every vertex $v \subseteq V$ is either in $S$ or adjacent to a vertex of $S$. A vertex of $S$ is said to dominate itself and all adjacent vertices. Each vertex is called a dominator, if it is in the dominating set, otherwise it is called a dominatee. That is, each dominatee is dominated by at least one dominator. A minimum DS (MDS) is a DS with the minimum cardinality. A dominating set is also an independent dominating set, if no two vertices in the set are adjacent. A CDS $S$ of a given graph $G$ is a dominating set whose induced sub-graph, denoted $\langle S\rangle$, is connected, and a MCDS is a CDS with the minimum cardinality. A MCDS forms a virtual backbone in the graph by which the routing overhead can be significantly reduced. The MDS and MCDS problems have been shown to be NP-Hard, ${ }^{30,31}$ and even for a unit disk graph (UDG), the problem of finding a MCDS is still NP-Hard. ${ }^{31}$

The closed neighborhood $N_{G}[v]$ of a vertex $v$ in graph $G$ consists of the vertices adjacent to $v$ and vertex $v$ itself. The closed neighborhood $N_{G}[S]$ of the set $S$ is the union $\bigcup_{v \in S} N_{G}[v]$. The subscript $G$ can be omitted if the meaning is clear from the context. A dominating set $S$ is a WCDS of a graph $G$, if the graph $\langle S\rangle_{W}=(N[S], E \cap(N[S] \times S))$ is a connected sub-graph of $G$. In other words, the weakly induced sub-graph $\langle S\rangle_{W}$ contains the vertices of $S$ and all vertices of $G$ which have at least one endpoint in $S$. Finding the minimum WCDS in the UDG is a NP-Hard problem ${ }^{2,26}$ and is one of the most investigated methods for cluster formation, in which a dominator node assumes the role of a cluster-head and its one-hop neighbors are assumed to be cluster members. A sample UDG and one of its WCDS are shown in Figs. 1(A) and 1(B), respectively. The dominator nodes assume the role of the cluster-heads and they have been colored black.

\subsection{Stochastic graph}

A vertex-weighted graph can be described by a triple $G=\langle\mathbf{V}, \mathbf{E}, W\rangle$, where $V=$ $\left\{v_{1}, v_{2}, \ldots, v_{n}\right\}$ denotes the vertex set, $\mathbf{E} \subseteq \mathbf{V} \times \mathbf{V}=\left\{e_{1}, e_{2}, \ldots, e_{m}\right\}$ denotes the 
edge set and $W=\left\{w_{1}, w_{2}, \ldots, w_{n}\right\}$ denotes the set of vertex weights such that $W\left(v_{i}\right)=w_{i}$, for all $i \in\{1,2, \ldots, n\}$. In most scenarios, the weight of the vertices is assumed to be fixed, but this is not always true and it varies with time. A vertexweighted graph is a stochastic vertex-weighted graph, and hereafter in this paper is called stochastic graph, if the weight of vertex $v_{i}$, for all $i \in\{1,2, \ldots, n\}$, is a random variable with probability distribution function $w_{i}$. Let $G=\langle\mathbf{V}, \mathbf{E}, W\rangle$ and $S=\left\{\delta_{1}, \delta_{2}, \ldots, \delta_{r}\right\}$ be a stochastic graph and the set of all its WCDSs, respectively. If $\bar{W}\left(v_{i}\right)$ and $\bar{W}\left(\delta_{i}\right)=\sum_{v_{i} \in \delta_{i}} \bar{W}\left(v_{i}\right)$ denote the expected weight of vertex $v_{i}$ and the expected weight of the WCDS $\delta_{i}$, respectively, therefore the stochastic WCDS $\delta^{*} \in S$ of graph $G$ is minimum, if and only if $\bar{W}\left(\delta^{*}\right)=\min _{\forall \delta_{i} \in S} \bar{W}\left(\delta_{i}\right)$. That is, the minimum WCDS of a given stochastic graph $G$ is defined as the stochastic WCDS with the minimum expected weight.

\subsection{Learning automata}

A learning automaton ${ }^{32-38}$ is an adaptive decision-making unit that improves its performance by learning how to choose the optimal action from a finite set of allowed actions through repeated interactions with a random environment. The action is chosen at random based on a probability distribution kept over the action set and at each instant the given action is served as the input to the random environment. The environment responds the taken action in turn with a reinforcement signal. The action probability vector is updated based on the reinforcement feedback from the environment. The objective of a learning automaton is to find the optimal action from the action set so that the average penalty received from the environment is minimized. ${ }^{32}$

The environment can be described by a triple $E \equiv\{\alpha, \beta, c\}$, where $\alpha \equiv$ $\left\{\alpha_{1}, \alpha_{2}, \ldots, \alpha_{r}\right\}$ represents the finite set of the inputs, $\beta \equiv\left\{\beta_{1}, \beta_{2}, \ldots, \beta_{m}\right\}$ denotes the set of the values can be taken by the reinforcement signal, and $c \equiv\left\{c_{1}, c_{2}, \ldots, c_{r}\right\}$ denotes the set of the penalty probabilities, where the element $c_{i}$ is associated with the given action $\alpha_{i}$. If the penalty probabilities are constant, the random environment is said to be a stationary random environment, and if they vary with time, the environment is called a non stationary environment. The environments depending on the nature of the reinforcement signal $\underline{\beta}$ can be classified into $P$-model, $Q$-model and $S$-model. The environments in which the reinforcement signal can only take two binary values 0 and 1 are referred to as $P$-model environments. Another class of the environment allows a finite number of the values in the interval $[0,1]$ can be taken by the reinforcement signal. Such an environment is referred to as $Q$-model environment. In $S$-model environments, the reinforcement signal lies in the interval $[a, b]$. The relationship between the learning automaton and its random environment has been shown in Fig. 2.

Learning automata can be classified into two main families: ${ }^{32-37}$ fixed structure learning automata and variable structure learning automata. Variable structure learning automata are represented by a triple $\langle\underline{\beta}, \underline{\alpha}, T\rangle$, where $\underline{\beta}$ is the set of inputs, 


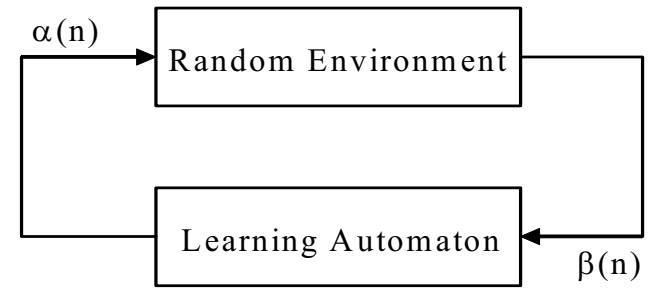

Fig. 2. The relationship between the learning automaton and its random environment.

$\underline{\alpha}$ is the set of actions, and $T$ is learning algorithm. The learning algorithm is a recurrence relation which is used to modify the action probability vector. Let $\alpha(k)$ and $\underline{p}(k)$ denote the action chosen at instant $k$ and the action probability vector on which the chosen action is based, respectively. The recurrence equation shown by (1) and (2) is a linear learning algorithm by which the action probability vector $\underline{p}$ is updated. Let $\alpha_{i}(k)$ be the action chosen by the automaton at instant $k$.

$$
p_{j}(n+1)= \begin{cases}p_{j}(n)+a\left[1-p_{j}(n)\right] & j=i \\ (1-a) p_{j}(n) & \forall j j \neq i\end{cases}
$$

When the taken action is rewarded by the environment (i.e., $\beta(n)=0)$ and

$$
p_{j}(n+1)= \begin{cases}(1-b) p_{j}(n) & j=i \\ (b / r-1)+(1-b) p_{j}(n) & \forall j j \neq i\end{cases}
$$

When the taken action is penalized by the environment (i.e., $\beta(n)=1) . r$ is the number of actions can be chosen by the automaton, $a(k)$ and $b(k)$ denote the reward and penalty parameters and determine the amount of increases and decreases of the action probabilities, respectively. If $a(k)=b(k)$, the recurrence equations (1) and (2) are called linear reward-penalty $\left(L_{R-P}\right)$ algorithm, if $a(k) \gg b(k)$ the given equations are called linear reward- $\varepsilon$ penalty $\left(L_{R-\varepsilon P}\right)$, and finally if $b(k)=0$ they are called linear reward-Inaction $\left(L_{R-I}\right)$. In the latter case, the action probability vectors remain unchanged when the taken action is penalized by the environment.

Learning automata have been found to be useful in systems where incomplete information about the environment, wherein the system operates, exists. Learning automata are also proved to perform well in dynamic environments. It has been shown in Refs. 39, 40-42 that the learning automata are capable of solving the NP-hard problems. Recently, several learning automata-based protocols have been designed for improving the performance of the wireless ad hoc networks. ${ }^{43,44-47}$

\subsubsection{Distributed learning automata}

A distributed learning automata (DLA) ${ }^{38}$ is a network of the learning automata which collectively cooperate to solve a particular problem. Formally, a DLA can be 


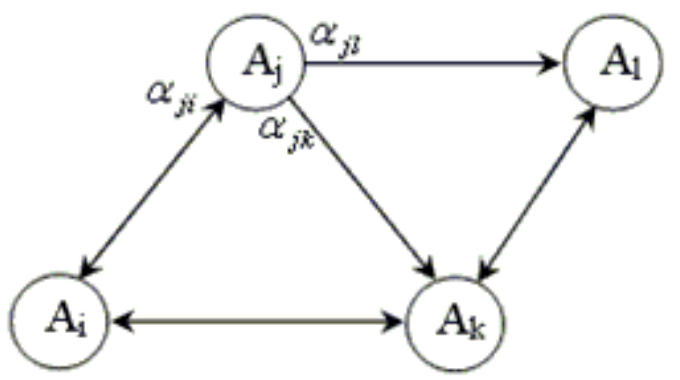

Fig. 3. Distributed learning automata.

defined by a quadruple $\left\langle A, E, T, A_{0}\right\rangle$, where $A=\left\{A_{1}, \ldots, A_{n}\right\}$ is the set of learning automata, $E \subset A \times A$ is the set of the vertices in which vertex $e_{(i, j)}$ corresponds to the action $\alpha_{j}$ of the automaton $A_{i}, T$ is the set of learning schemes with which the learning automata update their action probability vectors, and $A_{0}$ is the root automaton of DLA from which the automaton activation is started. An example of a DLA has been shown in Fig. 3 .

The operation of a DLA can be described as follows: At first, the root automaton randomly chooses one of its outgoing vertices (actions) according to its action probabilities and activates the learning automaton at the other end of the selected vertex. The activated automaton also randomly selects an action which results in activation of another automaton. The process of choosing the actions and activating the automata is continued until a leaf automaton (an automaton which interacts to the environment) is reached. The chosen actions, along the path induced by the activated automata between the root and leaf, are applied to the random environment. The environment evaluates the applied actions and emits a reinforcement signal to the DLA. The activated learning automata along the chosen path update their action probability vectors on the basis of the reinforcement signal by using the learning schemes. The paths from the unique root automaton to one of the leaf automata are selected until the probability with which one of the paths is chosen is close enough to unity. Each DLA has exactly one root automaton which is always activated, and at least one leaf automaton which is activated probabilistically.

\subsubsection{Variable action set learning automata}

A variable action set learning automaton is an automaton in which the number of actions available at each instant changes with time. It has been shown in Ref. 34 that a learning automaton with a changing number of actions is absolutely expedient and also $\varepsilon$-optimal, when the reinforcement scheme is $L_{R-I}$. Such an automaton has a finite set of $n$ actions, $\alpha=\left\{\alpha_{1}, \alpha_{2}, \ldots, \alpha_{n}\right\} . A=\left\{A_{1}, A_{2}, \ldots, A_{m}\right\}$ denotes the set of action subsets and $A(k) \subseteq \alpha$ is the subset of all the actions can be chosen by the learning automaton, at each instant $k$. The selection of the particular action subsets 
is randomly made by an external agency according to the probability distribution $q(k)=\left\{q_{1}(k), q_{2}(k), \ldots, q_{m}(k)\right\}$ defined over the possible subsets of the actions, where $q_{i}(k)=\operatorname{prob}\left[A(k)=A_{i} \mid A_{i} \in A, 1 \leq i \leq 2^{n}-1\right]$.

$\hat{p}_{i}(k)=\operatorname{prob}\left[\alpha(k)=\alpha_{i} \mid A(k), \alpha_{i} \in A(k)\right]$ is the probability of choosing action $\alpha_{i}$, conditioned on the event that the action subset $A(k)$ has already been selected and also $\alpha_{i} \in A(k)$. The scaled probability $\hat{p}_{i}(k)$ is defined as

$$
\hat{p}_{i}(k)=p_{i}(k) / K(k)
$$

where $K(k)=\sum_{\alpha_{i} \in A(k)} p_{i}(k)$ is the sum of the probabilities of the actions in subset $A(k)$, and $p_{i}(k)=\operatorname{prob}\left[\alpha(k)=\alpha_{i}\right]$.

The procedure of choosing an action and updating the action probabilities in a variable action set learning automaton can be described as follows. Let $A(k)$ be the action subset selected at instant $k$. Before choosing an action, the probabilities of all the actions in the selected subset are scaled as defined in Eq. (3). The automaton then randomly selects one of its possible actions according to the scaled action probability vector $\hat{p}(k)$. Depending on the response received from the environment, the learning automaton updates its scaled action probability vector. Note that the probability of the available actions is only updated. Finally, the probability vector of the actions of the chosen subset is rescaled as $p_{i}(k+1)=\hat{p}_{i}(k+1) \cdot K(k)$, for all $\alpha_{i} \in A(k)$. The absolute expediency and $\varepsilon$-optimality of the method described above have been proved in Ref. 34 .

\section{The Proposed Algorithms}

In this section, several learning automata-based approximation algorithms are proposed for solving the minimum WCDS problem in stochastic graphs. In the proposed algorithms, the vertex-weighted stochastic graph $G\langle V, E, W\rangle$ is served as the input of algorithm and the output is the WCDS of $G$. The reinforcement scheme by which the proposed algorithms update the probability vectors is $L_{R-I}$. It is assumed that the weight associated with each vertex of the stochastic graph $G$ is a random variable with unknown probability distribution function (PDF). Therefore, the PDF of the random variables must be estimated by a statistical method. The proposed algorithms taking advantage of learning automata are able to precisely determine the weight distribution of the vertices with a small number of samples. In these algorithms, a network of the learning automata isomorphic to the stochastic graph $G$ is initially formed by assigning to each vertex $v_{i}$ of the graph learning automaton $A_{i}$. The resultant network of learning automata can be described by a triple $\langle\underline{A}, \underline{\alpha}, \underline{W}\rangle$, where $\underline{A}=\left\{A_{1}, A_{2}, \ldots, A_{n}\right\}$ denotes the set of the learning automata, $\underline{\alpha}=\left\{\underline{\alpha}_{1}, \underline{\alpha}_{2}, \ldots, \underline{\alpha}_{n}\right\}$ denotes the set of actions in which $\underline{\alpha}_{i}=\left\{\underline{\alpha}_{i}^{1}, \underline{\alpha}_{i}^{2}, \ldots, \underline{\alpha}_{i}^{r i}\right\}$ defines the set of actions that can be taken by learning automaton $A_{i}$, for each $\underline{\alpha}_{i} \in \underline{\alpha}$, and $\underline{W}=\left\{w_{1}, w_{2}, \ldots, w_{n}\right\}$ denotes the set of weights such that $w_{i}$ (for all $i \in\{1,2, \ldots, n\})$ is the random weight associated with automaton $A_{i}$.

In the first proposed algorithm, the action-set of each learning automaton $A_{i}$, referred to as $\underline{\alpha}_{i}$, contains two actions $\alpha_{i}^{0}$ and $\alpha_{i}^{1}$. Choosing action $\alpha_{i}^{0}$ by learning 
automaton $A_{i}$ declares vertex $v_{i}$ as a dominatee vertex and choosing action $\alpha_{i}^{1}$ declares it as a dominator vertex. The proposed algorithm consists of a number of stages, and at each stage the vertices which are selected as dominators are added to the set of vertices by which a WCDS may be formed in that stage. This algorithm iteratively constructs a number of WCDSs and updates the action probability vectors until it finds a near optimal solution to the minimum WCDS problem. Stage $k$ of the proposed algorithm is briefly described in the following steps:

\section{Step 1. WCDS formation}

While the cardinality of the dominatee set is less than the cardinality of vertex set $V$ do

For all learning automata do in parallel

Each automaton $A_{i}$ chooses one of its actions

If the chosen action declares vertex $v_{i}$ as a dominator, then

Vertex $v_{i}$ is added to the set of dominators

Vertex $v_{i}$ and all its neighbors are added to the dominatee set

The random weight of vertex $v_{i}$ is added to the weight of

dominator set

\section{Step 2. Comparing the weight of the constructed WCDS with a dynamic threshold}

Let us assume that WCDS $\delta_{i}$ is selected at stage $k$.

The average weight of the constructed WCDS $\delta_{i}$ until stage $k$ is computed as

$$
\bar{W}\left(\delta_{i}^{k}\right)=\frac{1}{k_{i}} \sum_{j=1}^{k_{i}} W\left(\delta_{i}(j)\right)
$$

where $k_{i}$ denotes the number of times the WCDS $\delta_{i}$ is constructed until stage $k$, and $W\left(\delta_{i}(j)\right)$ denotes the weight of the $j^{t h}$ sampled WCDS $\delta_{i}$ which is defined as

$$
W\left(\delta_{i}(j)\right)=\sum_{v_{s} \in \delta_{i}} W\left(v_{s}(j)\right)
$$

where $W\left(v_{s}(j)\right)$ denotes the weight of the vertex $v_{s}$ in the $j^{t h}$ sample taken from $\delta_{i}$. The average weight computed for the constructed WCDS $\delta_{i}$ is compared to the dynamic threshold $T_{k}$. At stage $k>1$, the value of the dynamic threshold $T_{k}$ is calculated as

$$
T_{k}=\frac{1}{r} \sum_{i=1}^{r} \bar{W}\left(\delta_{i}^{k}\right)
$$

where $r$ denotes the number of all WCDSs of $G$. 


\section{Step 3. Updating the action probability vectors}

Depending on the comparison result in step 2, all the learning automata (only the activated learning automata in Algorithm 5) reward their chosen actions if the average weight of the constructed WCDS $\delta_{i}$ is less than or equal to the dynamic threshold $T_{k-1}$, and penalize them otherwise. Each learning automaton then updates its action probability vector using $L_{R-I}$ reinforcement scheme.

\section{Step 4. Stopping Condition}

The process of constructing the WCDSs and updating the action probabilities are repeated until the product of the probability of choosing the vertices of the constructed WCDS is greater than a certain threshold $(P)$ or the number of constructed WCDS exceeds a pre-specified threshold $(K)$ The WCDS which is formed just before the algorithm stops is the WCDS with the minimum expected weight among all WCDSs of the stochastic graph $G$.

The proposed algorithm has been described in Fig. 4 in more detail.

In Algorithm 1, it is assumed that the number of actions that can be taken by each learning automaton is fixed and does not vary with time. Fixed action-set learning automaton used in Algorithm 1 significantly increases the number of vertices that must be sampled for constructing the WCDS (or the cardinality of the constructed WCDS) and hence increases the running time of algorithm dramatically. To alleviate this problem, we propose another algorithm called Algorithm 2 in which the number of actions available for each learning automaton changes at each stage. The action-set of Algorithm 2 is similar to that of Algorithm 1. In this algorithm, each learning automaton $A_{i}$ disables its action $\alpha_{i}^{1}$ (which declares vertex $v_{i}$ as a dominator vertex) as described in Subsection 2.3.2 on variable action-set learning automata, if one of its neighbors has declared itself as a dominator vertex earlier. The action-set remains unchanged otherwise. In this algorithm, the learning automata are randomly and sequentially activated and choose one of their available actions based on the action probability vector scaled after disabling the actions as given in Eq. (3). The action probabilities are then rescaled after evaluating (rewarding or penalizing) the constructed WCDS. This reduction in number of actions considerably reduces the cardinality of the constructed WCDS and so increases the speed of convergence to the minimum WCDS.

Since the first two proposed algorithms (Algorithms 1 and 2) can not guarantee to from a WCDS at each stage, the first step (i.e., WCDS formation step) of these algorithms needs to be repeated until a weakly connected dominating set is finally formed. This dramatically increases the running time of algorithm. To solve the above mentioned problem, we propose another learning automata-based algorithm in which a weakly connected dominating set can be constructed at each stage. This algorithm which is called Algorithm 3, is very similar to Algorithm 1, but differs in the action-set defined for each learning automaton as well as the way of constructing the WCDSs (step 1). In Algorithm 3, learning automaton $A_{j}$ (for all $j \in\{1,2, \ldots, n\})$ assigned to vertex $v_{j}$, where vertex $v_{j}$ is adjacent to vertex $v_{i}$, 


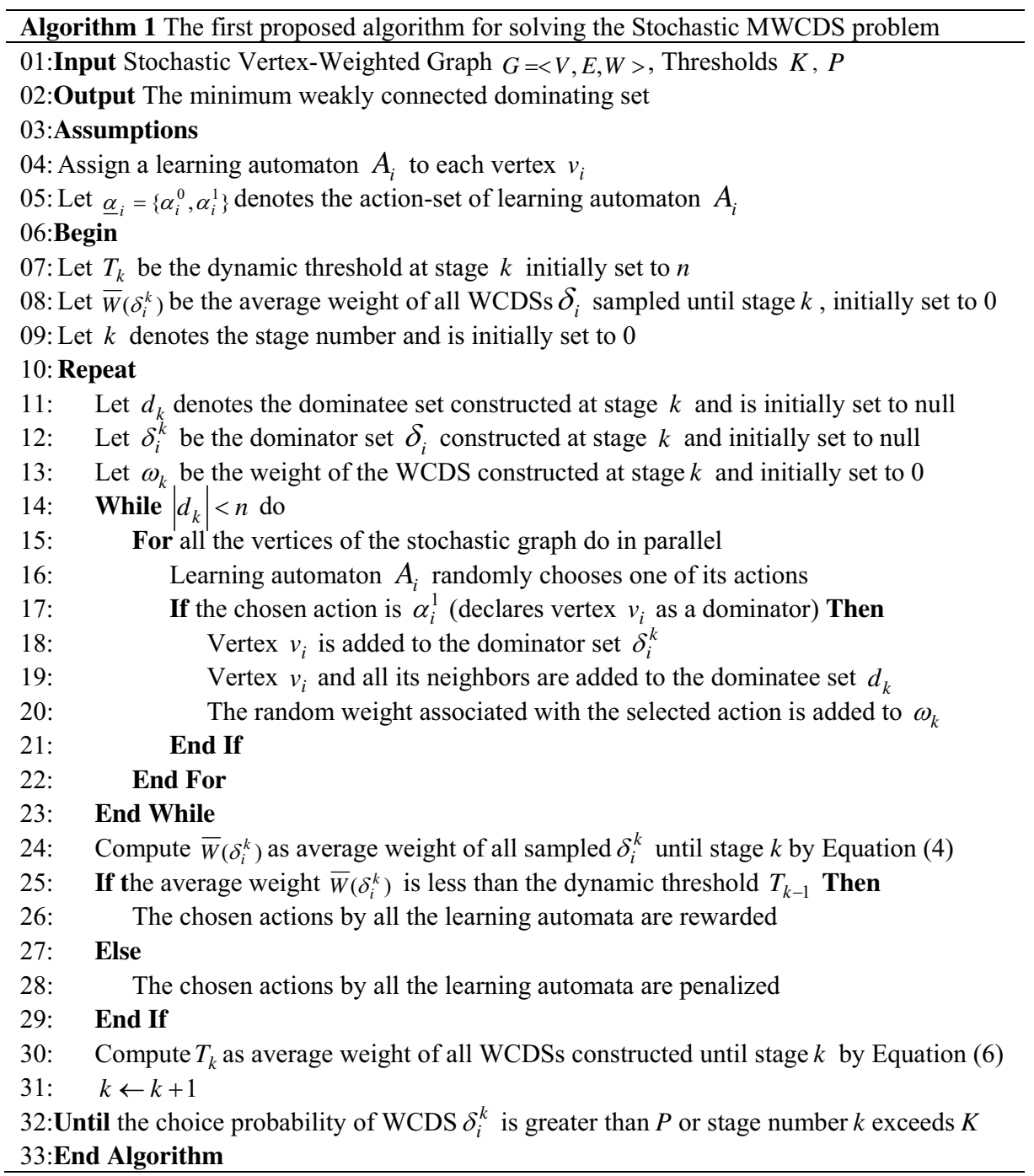

Fig. 4. Pseudo code of Algorithm 1.

as well as learning automaton $A_{i}$ itself, form the action-set of learning automaton $A_{i}$. That is, $\underline{\alpha}_{i}=\left\{A_{j} \mid \forall j ; j=i\right.$ or $\left.\left(v_{i}, v_{j}\right) \in E\right\}$. For each learning automaton $A_{i}$, choosing a given action $A_{j} \in \underline{\alpha}_{i}$ declares vertex $v_{j}$ (the vertex corresponding to the selected action) as its dominator. In other words, each learning automaton chooses a dominator for its corresponding vertex among its neighbors or declares itself as a dominator node. The dominators selected by all learning automata constitute the current WCDS. The step of WCDS formation of Algorithm 3 (step 1) at each stage $k$ is described as shown in Fig. 5. The remaining steps (steps 2, 3, and 4) of Algorithm 3 are the same as those described in Algorithm 1. 


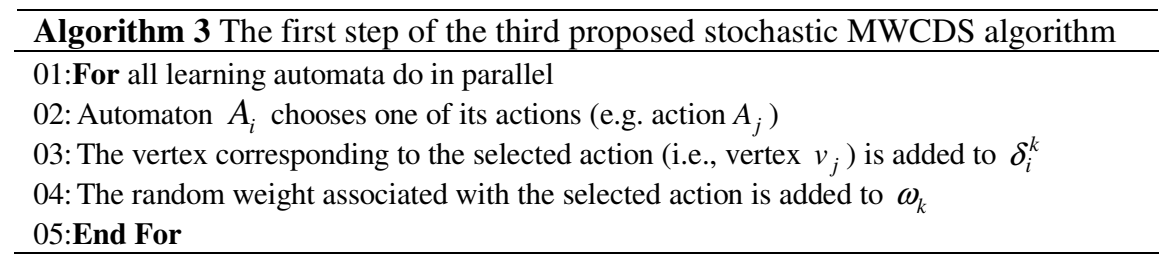

Fig. 5. The WCDS formation step in Algorithm 3.

The dominating set formation process of Algorithm 3 may result in redundant dominators in WCDS by which no more vertices can be dominated. This enlarges the size of WCDS and increases the sampling rate of algorithm too. As shown in Algorithm 2, the variable action-set learning automata enable algorithm to reduce the number of vertices needs to be sampled (sampling rate) for constructing the WCDS at each stage. The reduced sampling rate increases the convergence speed to the optimal solution. To achieve such a performance in Algorithm 3, we propose another algorithm in which the action-set of each learning automaton changes with time as follows:

If vertex $v_{i}$ is selected as a dominator, then learning automaton $A_{i}$ and all the learning automata which are adjacent to learning automaton $A_{i}$ retain the action corresponding to learning automaton $A_{i}$ if any, and disable all the other actions (if they have not been disabled yet) as described in Subsection 2.3.2. That is, when a given vertex $v_{i}$ (associated with automaton $A_{i}$ ) is selected as a dominator, the action-set of its neighboring learning automata is pruned in such a way that the dominator that can be chosen by learning automaton $A_{i}$ and its neighbors is vertex $v_{i}$ only. Such a pruned action-set avoids selecting the adjacent vertices as dominators, and so causes significantly smaller WCDSs. In this algorithm, the learning automata are sequentially activated at random, and each learning automaton chooses one of its possible actions according to its scaled action probability vector as described in Algorithm 2. The algorithm in which the WCDS is thus constructed is called Algorithm 4 .

The last algorithm we propose for approximating a near optimal solution to the stochastic minimum WCDS problem is based on distributed learning automata (DLA) described in Subsection 2.3.1 which is called Algorithm 5. This algorithm like Algorithm 3 differs from Algorithm 1 only in the action-set defined for each learning automaton as well as the method by which the WCDS is constructed at each stage. In this algorithm, the set of actions that can be taken by each learning automaton $A_{i}$ initially includes the set of all learning automata (i.e., $\underline{A}=\left\{A_{1}, A_{2}, \ldots, A_{n}\right\}$ ) except automata $A_{i}$ and its neighbors. That is, the action-set of learning automaton $A_{i}$ is defined as $\underline{\alpha}_{i}=\underline{A}-\left(\left\{A_{i}\right\} \cup\left\{A_{j} \mid \forall j ;\left(v_{i}, v_{j}\right) \in E\right\}\right)$. Each learning automaton can be in one of two states: active and passive. Each learning automaton is initially set to the passive state. The selected automata at each stage are activated and added to the set of dominator vertices. The WCDS formation step of Algorithm 5 


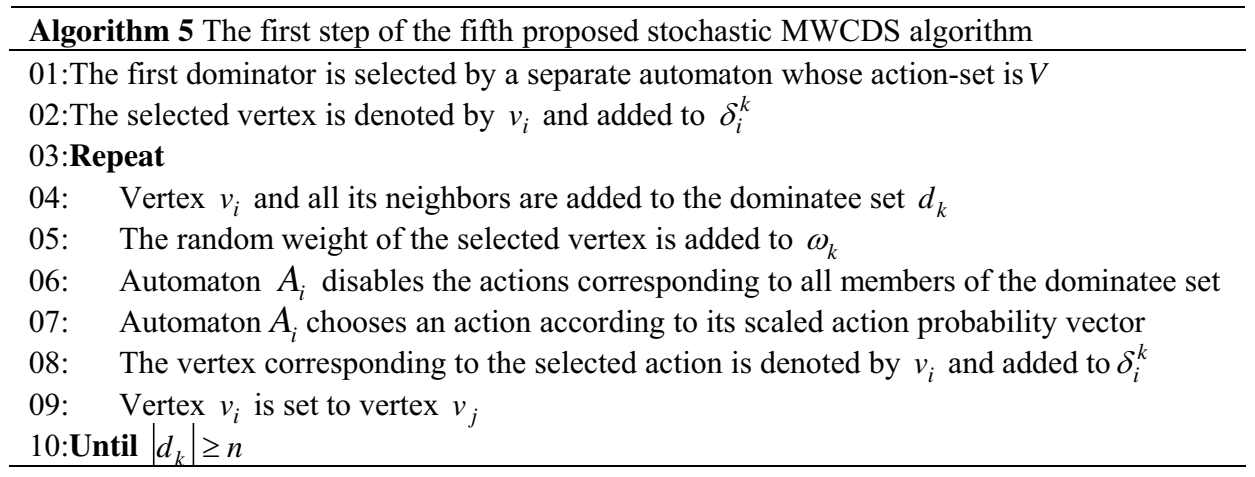

Fig. 6. The WCDS formation step in Algorithm 5.

at each stage $k$ is described as shown in Fig. 6. The remaining steps (steps 2, 3, and 4) of Algorithm 5 are the same as those described in Algorithm 1.

\section{Convergence Results}

In this section we prove two main results of the paper. The first result is concerned with the proof of the convergence of Algorithm 1 to the optimal solution. The method used to prove the convergence of the Algorithm 1 follows the method given in Refs. 32 and 36 to analysis the behavior and to prove the convergence of the learning automata operating in non-stationary environments. In addition to the convergence proof, it is also shown that by a proper choice of the learning rate $a$, the probability with which Algorithm 1 finds the optimal solution is as close to unity as possible where each learning automaton has a $L_{R-I}$ learning algorithm. The second result concerns the relationship between the convergence error parameter $\varepsilon$ (i.e., the error parameter involved in the SSM) and the learning rate of Algorithm 1. The second result we discuss in this paper demonstrates the existence of a learning rate under which the probability of constructing (or converging to) the WCDS with the minimum expected weight is larger than $1-\varepsilon$.

Theorem 1. Let $q_{i}(k)$ be the probability of constructing the $W C D S \omega_{i}$ in a given stochastic graph, at stage $k$. If $\underline{q}(k)$ evolves according to algorithm 1 , then for every $\varepsilon>0$, there exists a learning rate $a^{*}(\varepsilon) \in(0,1)$ such that for all $a \in\left(0, a^{*}\right)$, we have

$$
\operatorname{Prob}\left[\lim _{k \rightarrow \infty} q_{i}(k)=1\right] \geq 1-\varepsilon .
$$

Proof. The steps of the convergence proof are briefly outlined as follows. At first, it is proved that, the penalty probabilities of the WCDSs converge to the constant values of the final penalty probabilities, for large enough values of $k$ (Lemma 1). Then, it is shown that, the probability of choosing the WCDS with the minimum expected weight, is a sub-Martingale process for large values of $k$, and so the changes 
in the probability of constructing the minimum WCDS is always nonnegative (Lemmas 2 and 3). Finally, the convergence of the algorithm 1 to the minimum WCDS is proved by using Martingale convergence theorems. Therefore, the following lemmas need to be proved before stating the proof of the theorem.

Lemma 1. If the $W C D S \omega_{i}$ is penalized with probability $c_{i}(k)$ at stage $k$ (i.e., $\left.c_{i}(k)=\operatorname{prob}\left[\bar{W} \omega_{i}>T_{k}\right]\right)$, and $\operatorname{Lim}_{k \rightarrow \infty} c_{i}(k)=c_{i}{ }^{*}$, then for each $\varepsilon \in(0,1)$ and $k>K(\varepsilon)$ we have,

$$
\operatorname{prob}\left[\left|c_{i}^{*}-c_{i}(k)\right|>0\right]<\varepsilon .
$$

Proof. Let $c_{j}(k)$ (for all $j=1,2, \ldots, r$ ) denotes the probability with which the WCDS $\omega_{i}$ is penalized at stage $k, r$ denotes the number of all WCDSs of $G$, and $c_{j}{ }^{*}$ denotes the probability $c_{j}(k)$ for large values of $k$. Using weak law of large numbers, we conclude that

$$
\operatorname{Lim}_{k \rightarrow \infty} \operatorname{prob}\left[\left|c_{i}^{*}-c_{i}(k)\right|>\varepsilon\right] \rightarrow 0 .
$$

For every $\varepsilon \in(0,1)$, there exists a $a^{*}(\varepsilon) \in(0,1)$ and $K_{0}(\varepsilon)<\infty$ such that for all $a<a^{*}$ and $k>K_{0}$ we have, $\operatorname{Prob}\left[\left|c_{i}^{*}-c_{i}(k)\right|>0\right]<\varepsilon$, and the proof of Lemma 1 is completed.

Lemma 2. Let $c_{j}(k)=\operatorname{prob}\left[\bar{W} \omega_{j}(k+1)>T_{k}\right]$ and $d_{j}=1-c_{j}$ be the probability of penalizing and rewarding the $W C D S \omega_{j}$ (for all $j=1,2, \ldots, r$ ), respectively. If $\underline{q}(k)$ evolves according to algorithm 1 , then the conditional expectation of $q_{i}(k)$ is defined as

$E\left[q_{i}(k+1) \mid q(k)\right]=\sum_{j=1}^{r} q_{j}(k)\left[c_{j}(k) q_{i}(k)+d_{j}(k)\left(\prod_{v_{m} \notin \omega_{i}}\left(1-p_{1}^{m}(k)\right) \prod_{v_{m} \in \omega_{i}} p_{1}^{m}(k)\right)\right]$

where

$$
p_{1}^{m}(k+1)= \begin{cases}p_{1}^{m}(k)+a\left(1-p_{1}^{m}(k)\right) ; & v_{m} \in \omega_{j} \\ p_{1}^{m}(k) \cdot(1-a) ; & v_{m} \notin \omega_{j}\end{cases}
$$

where $p_{1}^{m}(k)$ is the probability that vertex $v_{m}$ declares itself as a dominator vertex, at stage $k$, and $v_{m} \in \omega_{i}$, if it declares itself as a dominator vertex (or chooses action $\left.\alpha_{m}^{1}\right)$ and $v_{m} \notin \omega_{i}$ otherwise.

Proof. Since the reinforcement scheme used to update the probability vectors in algorithm 1 is $L_{R-I}$, at each stage $k$, the probability of constructing the WCDS $\omega_{i}$ (i.e., $q_{i}(k)$ ), remains unchanged with probability $c_{j}(k)$ (for all $j=1,2, \ldots, r$ ), when the constructed WCDS $\omega_{j}$ is penalized by the random environment. On the other hand, when the constructed WCDS $\omega_{j}$ is rewarded, the probability of choosing the vertices of the WCDS $\omega_{i}$, which are in the constructed WCDS $\omega_{j}$ increases 

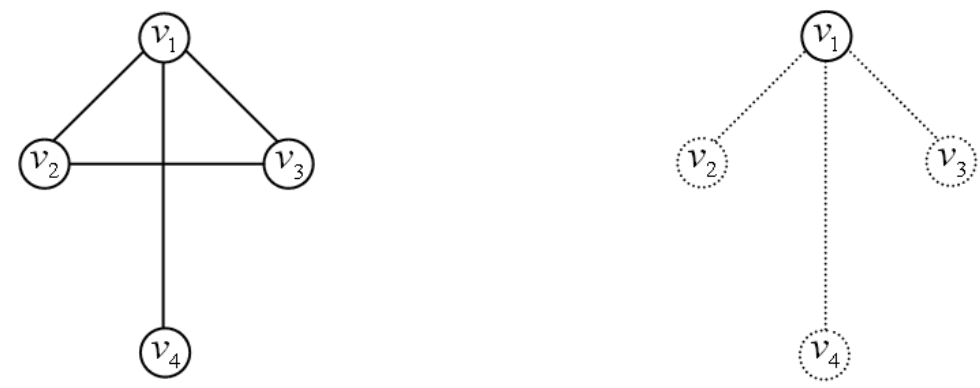

Fig. 7. Sample graph $G$ and its minimum WCDS.

by a given learning rate as that of the other vertices decreases. To illustrate the proof of the lemma in more details, we prove it for the minimum WCDS of the graph shown in Fig. 7. As shown in Fig. 7, graph $G$ has 4 vertices, 4 edges, and 11 WCDSs as follows: $\omega_{1}=\left\{v_{1}\right\}, \omega_{2}=\left\{v_{1}, v_{2}\right\}, \omega_{3}=\left\{v_{1}, v_{3}\right\}, \omega_{4}=\left\{v_{1}, v_{4}\right\}$, $\omega_{5}=\left\{v_{2}, v_{4}\right\}, \omega_{6}=\left\{v_{3}, v_{4}\right\}, \omega_{7}=\left\{v_{1}, v_{2}, v_{3}\right\}, \omega_{8}=\left\{v_{1}, v_{2}, v_{4}\right\}, \omega_{9}=\left\{v_{1}, v_{3}, v_{4}\right\}$, $\omega_{10}=\left\{v_{2}, v_{3}, v_{4}\right\}, \omega_{11}=\left\{v_{1}, v_{2}, v_{3}, v_{4}\right\}$. It is also assumed that $\omega_{1}$ is the WCDS with the minimum expected weight of the graph given in Fig. 7 .

Let

$$
q_{i}(k)=\prod_{v_{m} \notin \omega_{i}} p_{0}^{m}(k) \prod_{v_{m} \in \omega_{i}} p_{1}^{m}(k)
$$

be the probability of constructing the WCDS $\omega_{i}$ at stage $k$, where $p_{0}^{m}$ and $p_{1}^{m}$ denote the probability with which vertex $v_{m}$ declares itself as a dominatee vertex (or chooses action $\alpha_{m}^{0}$ ) and as a dominator vertex (or chooses action $\alpha_{m}^{1}$ ), respectively, and $v_{m} \in \omega_{i}$, if it declares itself as a dominator vertex and $v_{m} \notin \omega_{i}$ otherwise. Therefore, for the graph shown in Fig. 3 we have

$$
\begin{aligned}
q_{1}(k) & =p_{1}^{1}(k) p_{0}^{2}(k) p_{0}^{3}(k) p_{0}^{4}(k) \\
q_{2}(k) & =p_{1}^{1}(k) p_{1}^{2}(k) p_{0}^{3}(k) p_{0}^{4}(k) \\
q_{3}(k) & =p_{1}^{1}(k) p_{0}^{2}(k) p_{1}^{3}(k) p_{0}^{4}(k) \\
q_{4}(k) & =p_{1}^{1}(k) p_{0}^{2}(k) p_{0}^{3}(k) p_{1}^{4}(k) \\
q_{5}(k) & =p_{0}^{1}(k) p_{1}^{2}(k) p_{0}^{3}(k) p_{1}^{4}(k) \\
q_{6}(k) & =p_{0}^{1}(k) p_{0}^{2}(k) p_{1}^{3}(k) p_{1}^{4}(k) \\
q_{7}(k) & =p_{1}^{1}(k) p_{1}^{2}(k) p_{1}^{3}(k) p_{0}^{4}(k) \\
q_{8}(k) & =p_{1}^{1}(k) p_{1}^{2}(k) p_{0}^{3}(k) p_{1}^{4}(k) \\
q_{9}(k) & =p_{1}^{1}(k) p_{0}^{2}(k) p_{1}^{3}(k) p_{1}^{4}(k) \\
q_{10}(k) & =p_{0}^{1}(k) p_{1}^{2}(k) p_{1}^{3}(k) p_{1}^{4}(k) \\
q_{11}(k) & =p_{1}^{1}(k) p_{1}^{2}(k) p_{1}^{3}(k) p_{1}^{4}(k) .
\end{aligned}
$$


The conditional expectation of $q_{1}(k+1)$, assuming $\underline{q}(k)$ evolves according to algorithm 1, is defined as

$$
\begin{aligned}
& E\left[q_{1}(k+1) \mid q(k)\right]=q_{1}(k)\left[c_{1} q_{1}(k)+d_{1}(k)\left\{p_{1}^{1}(k)+a\left(1-p_{1}^{1}(k)\right)\right\}\right. \\
& \left.\left\{p_{0}^{2}(k)+a\left(1-p_{0}^{2}(k)\right)\right\}\left\{p_{0}^{3}(k)+a\left(1-p_{0}^{3}(k)\right)\right\}+\left\{p_{0}^{4}(k)+a\left(1-p_{0}^{4}(k)\right)\right\}\right] \\
& +q_{2}(k)\left[c_{2} q_{1}(k)+d_{2}(k)\left\{p_{1}^{1}(k)+a\left(1-p_{1}^{1}(k)\right)\right\}\left\{p_{0}^{2}(k)(1-a)\right\}\left\{p_{0}^{3}(k)+a\left(1-p_{0}^{3}(k)\right)\right\}\right. \\
& \left.\left\{p_{0}^{4}(k)+a\left(1-p_{0}^{4}(k)\right)\right\}\right]+q_{3}(k)\left[c_{3} q_{1}(k)+d_{3}(k)\left\{p_{1}^{1}(k)+a\left(1-p_{1}^{1}(k)\right)\right\}\right. \\
& \left.\left\{p_{0}^{2}(k)+a\left(1-p_{0}^{2}(k)\right)\right\}\left\{p_{0}^{3}(k)(1-a)\right\}\left\{p_{0}^{4}(k)+a\left(1-p_{0}^{4}(k)\right)\right\}\right]+q_{4}(k)\left[c_{4} q_{1}(k)\right. \\
& +d_{4}(k)\left\{p_{1}^{1}(k)+a\left(1-p_{1}^{1}(k)\right)\right\}\left\{p_{0}^{2}(k)+a\left(1-p_{0}^{2}(k)\right)\right\}\left\{p_{0}^{3}(k)+a\left(1-p_{0}^{3}(k)\right)\right\} \\
& \left.\left\{p_{0}^{4}(k)(1-a)\right\}\right]+q_{5}(k)\left[c_{5} q_{1}(k)+d_{5}(k)\left\{p_{1}^{1}(k)(1-a)\right\}\left\{p_{0}^{2}(k)(1-a)\right\}\right. \\
& \left.\left\{p_{0}^{3}(k)+a\left(1-p_{0}^{3}(k)\right)\right\}\left\{p_{0}^{4}(k)(1-a)\right\}\right]+q_{6}(k)\left[c_{6} q_{1}(k)+d_{6}(k)\left\{p_{1}^{1}(k)(1-a)\right\}\right. \\
& \left.\left\{p_{0}^{2}(k)+a\left(1-p_{0}^{2}(k)\right)\right\}\left\{p_{0}^{3}(k)(1-a)\right\}\left\{p_{0}^{4}(k)(1-a)\right\}\right]+q_{7}(k)\left[c_{7} q_{1}(k)\right. \\
& \left.+d_{7}(k)\left\{p_{1}^{1}(k)+a\left(1-p_{1}^{1}(k)\right)\right\}\left\{p_{0}^{2}(k)(1-a)\right\}\left\{p_{0}^{3}(k)(1-a)\right\}\left\{p_{0}^{4}(k)+a\left(1-p_{0}^{4}(k)\right)\right\}\right] \\
& +q_{8}(k)\left[c_{8} q_{1}(k)+d_{8}(k)\left\{p_{1}^{1}(k)+a\left(1-p_{1}^{1}(k)\right)\right\}\left\{p_{0}^{2}(k)(1-a)\right\}\left\{p_{0}^{3}(k)+a\left(1-p_{0}^{3}(k)\right)\right\}\right. \\
& \left.\left\{p_{0}^{4}(k)(1-a)\right\}\right]+q_{9}(k)\left[c_{9} q_{1}(k)+d_{9}(k)\left\{p_{1}^{1}(k)+a\left(1-p_{1}^{1}(k)\right)\right\}\right. \\
& \left.\left\{p_{0}^{2}(k)+a\left(1-p_{0}^{2}(k)\right)\right\}\left\{p_{0}^{3}(k)(1-a)\right\}\left\{p_{0}^{4}(k)(1-a)\right\}\right]+q_{10}(k)\left[c_{10} q_{1}(k)\right. \\
& \left.+d_{10}(k)\left\{p_{1}^{1}(k)(1-a)\right\}\left\{p_{0}^{2}(k)(1-a)\right\}\left\{p_{0}^{3}(k)(1-a)\right\}\left\{p_{0}^{4}(k)(1-a)\right\}\right] \\
& +q_{11}(k)\left[c_{11} q_{1}(k)+d_{11}(k)\left\{p_{1}^{1}(k)+a\left(1-p_{1}^{1}(k)\right)\right\}\right. \\
& \left.\left\{p_{0}^{2}(k)(1-a)\right\}\left\{p_{0}^{3}(k)(1-a)\right\}\left\{p_{0}^{4}(k)(1-a)\right\}\right] .
\end{aligned}
$$

After simplifying all the terms in the right hand side of the equation above, and also some algebraic manipulations, we obtain

$$
E\left[q_{1}(k+1) \mid q(k)\right]=\sum_{j=1}^{11} q_{j}(k)\left[c_{j}(k) q_{1}(k)+d_{j}(k)\left(\prod_{v_{m} \notin \omega_{1}} p_{0}^{m}(k) \prod_{v_{m} \in \omega_{1}} p_{1}^{m}(k)\right)\right]
$$

where

$$
\begin{aligned}
& p_{0}^{m}(k+1)= \begin{cases}p_{0}^{m}(k)+a\left(1-p_{0}^{m}(k)\right) ; & v_{m} \notin \omega_{j} \\
p_{0}^{m}(k) \cdot(1-a) ; & v_{m} \in \omega_{j}\end{cases} \\
& p_{1}^{m}(k+1)= \begin{cases}p_{1}^{m}(k)+a\left(1-p_{1}^{m}(k)\right) ; & v_{m} \in \omega_{j} \\
p_{1}^{m}(k) \cdot(1-a) ; & v_{m} \notin \omega_{j}\end{cases}
\end{aligned}
$$

where $p_{1}^{m}(k)$ is the probability that vertex $v_{m}$ declares itself as a dominator vertex, at stage $k$. Since each learning automaton has only two possible actions, the probability with which vertex $v_{m}$ declares itself as a dominatee vertex is defined as $1-p_{1}^{m}(k)$. Hence, $q_{i}$ at stage $k$ can be defined as

$$
\prod_{v_{m} \notin \omega_{i}}\left(1-p_{1}^{m}(k)\right) \prod_{v_{m} \in \omega_{i}} p_{1}^{m}(k)
$$


and so we have

$$
E\left[q_{1}(k+1) \mid(q(k)]=\sum_{j=1}^{11} q_{j}(k)\left[c_{j}(k) q_{1}(k)+d_{j}(k)\left(\prod_{v_{m} \notin \omega_{i}}\left(1-p_{1}^{m}(k)\right) \prod_{v_{m} \in \omega_{i}} p_{1}^{m}(k)\right)\right]\right.
$$

where

$$
p_{1}^{m}(k+1)= \begin{cases}p_{1}^{m}(k)+a\left(1-p_{1}^{m}(k)\right) ; & v_{m} \in \omega_{j} \\ p_{1}^{m}(k) \cdot(1-a) ; & v_{m} \notin \omega_{j}\end{cases}
$$

and hence the proof of the lemma.

Lemma 3. Let $q_{i}(k)$ denotes the probability of formation of the minimum WCDS $\omega_{i}$, at stage $k$. The increment in the conditional expectation of $q_{i}(k)$ is always nonnegative, assuming $\underline{q}(k)$ is updated according to algorithm 1. That is, $\Delta q_{i}(k)>0$.

Proof. Define

$$
\Delta q_{i}(k)=E\left[q_{i}(k+1) \mid q(k)\right]-q_{i}(k) .
$$

From Lemma 2, we have

$$
\Delta q_{i}(k)=\sum_{j=1}^{r} q_{j}(k)\left[c_{j}(k) q_{i}(k)+d_{j}(k)\left(\prod_{v_{m} \notin \omega_{i}}\left(1-p_{1}^{m}(k)\right) \prod_{v_{m} \in \omega_{i}} p_{1}^{m}(k)\right)\right]-q_{i}(k)
$$

where

$$
p_{1}^{m}(k+1)= \begin{cases}p_{1}^{m}(k)+a\left(1-p_{1}^{m}(k)\right) ; & v_{m} \in \omega_{j} \\ p_{1}^{m}(k) \cdot(1-a) ; & v_{m} \notin \omega_{j} .\end{cases}
$$

Let

$$
\prod_{\substack{\omega_{i} \\ n \in\{0,1\}}} \pi_{n}^{m}(k)=\prod_{v_{m} \notin \omega_{i}}\left(1-p_{1}^{m}(k)\right) \prod_{v_{m} \in \omega_{i}} p_{1}^{m}(k),
$$

for notational convenience. Therefore, we have

$$
\Delta q_{i}(k)=\sum_{j=1}^{11} q_{j}(k)\left[c_{j}(k) q_{i}(k)+d_{j}(k) \prod_{\substack{\omega_{i} \\ n \in\{0,1\}}} \pi_{n}^{m}(k)\right]-q_{i}(k)
$$

where

$$
\pi_{n}^{m}(k+1)= \begin{cases}p_{n}^{m}(k)+a\left(1-p_{n}^{m}(k)\right) ; & \left(v_{m} \notin \omega_{j}, n=0\right) \text { or }\left(v_{m} \in \omega_{j}, n=1\right) \\ p_{n}^{m}(k) \cdot(1-a) ; & \left(v_{m} \notin \omega_{j}, n=1\right) \text { or }\left(v_{m} \in \omega_{j}, n=0\right)\end{cases}
$$

where $\pi_{n}^{m}(k)$ is the probability with which vertex $v_{m}$, at stage $k$, declares itself as a dominator vertex, when $n=1$, and as a dominatee vertex otherwise.

Since the probability that the WCDS is constructed, rewarded or penalized, is defined as the product of the probability of declaring the vertices in the constructed 
WCDS as dominators or dominatees as defined in Eq. (10), we have

$$
\begin{aligned}
\Delta q_{i}(k)= & \sum_{j=1}^{r} \prod_{\substack{\omega_{j} \\
n \in\{0,1\}}} p_{n}^{m}(k)\left[\prod_{\substack{\omega_{j} \\
n \in\{0,1\}}} c_{n}^{m}(k) \prod_{\substack{\omega_{i} \\
n \in\{0,1\}}} p_{n}^{m}(k)+\prod_{\substack{\omega_{j} \\
n \in\{0,1\}}} d_{n}^{m}(k) \prod_{\substack{\omega_{i} \\
n \in\{0,1\}}} \pi_{n}^{m}(k)\right] \\
& -\prod_{\substack{\omega_{i} \\
n \in\{0,1\}}} p_{n}^{m}(k)
\end{aligned}
$$

where $\pi_{n}^{m}(k)$ is defined as given in Eq. $(11), c_{n}^{m}(k)$ is the probability of penalizing the action chosen by vertex $v_{m}$ at stage $k$, and $d_{n}^{m}(k)=1-c_{n}^{m}(k)$.

$$
\Delta q_{i}(k)=\prod_{\substack{\omega_{j} \\ n \in\{0,1\}}} E\left[p_{n}^{m}(k+1) \mid p^{m}(k)\right]-\prod_{\substack{\omega_{j} \\ n \in\{0,1\}}} p_{n}^{m}(k) .
$$

The equality above can be rewritten as

$$
\Delta q_{i}(k) \geq \prod_{\substack{\omega_{j} \\ n \in\{0,1\}}}\left(E\left[p_{n}^{m}(k+1) \mid p^{m}(k)\right]-p_{n}^{m}(k)\right)=\prod_{\substack{\omega_{j} \\ n \in\{0,1\}}} \Delta p_{n}^{m}(k)
$$

and

$$
\underset{n \in\{0,1\}}{\Delta p_{n}^{m}(k)}=a \cdot p_{n}^{m}(k) \sum_{s \neq n} p_{s}^{m}(k) \cdot\left(c_{s}^{m}(k)-c_{n}^{m}(k)\right)
$$

$q_{i}(k) \in(0,1)$ for all $\underline{q} \in S_{r}^{0}$, where $S_{r}=\left\{\underline{q}(k): 0 \leq q_{i}(k) \leq 1 ; \sum_{i=1}^{r} q_{i}(k)=1\right\}$ and $S_{r}^{0}$ denotes the interior of $S_{r}$. Hence, $p_{n}^{\bar{m}}(k) \in(0,1)$ for all $m, n$. Since action $\alpha_{n}^{m}$ is the action with the minimum penalty probability which can be selected by automaton $A_{m}$, it is shown that $c_{s}^{m^{*}}-c_{n}^{m^{*}}>0$, for all $s \neq n$, where $c_{s}^{m^{*}}$ is the final value to which the penalty probability $c_{s}^{m^{*}}$ is converged. It follows from Lemma 1 that for large values of $k, c_{s}^{m}(k)-c_{n}^{m}(k)>0$. Therefore, we conclude that for large values of $k$, the right hand side of the equation above consists of the nonnegative quantities, and so we have

$$
\prod_{\substack{\omega_{i} \\ n \in\{0,1\}}} a \cdot p_{n}^{m}(k) \sum_{s \neq n} p_{s}^{m}(k) \cdot\left(c_{s}^{m}(k)-c_{n}^{m}(k)\right) \geq 0
$$

and from Eq. (12), we have

$$
\Delta q_{i}(k) \geq \prod_{\substack{\omega_{i} \\ n \in\{0,1\}}} a \cdot p_{n}^{m}(k) \sum_{s \neq n} p_{s}^{m}(k) \cdot\left(c_{s}^{m}(k)-c_{n}^{m}(k)\right)
$$

which completes the proof of this lemma.

Corollary 1. The set of unit vectors in $S_{r}-S_{r}^{0}$, where $S_{r}^{0}=\left\{\underline{q}(k): q_{i}(k) \in(0,1)\right.$; $\left.\sum_{i=1}^{r} q_{i}(k)=1\right\}$, forms the set of all absorbing barriers of the markov process $\{\underline{q}(k)\}_{k \geq 1}$. 
Proof. Lemma 3 implicitly proves that $\{q(k)\}$ is a sub-Martingale. Using Martingale theorems, and the fact that $\{q(k)\}$ is non-negative and uniformly bounded, it is concluded that $\operatorname{Lim}_{k \rightarrow \infty} q_{i}(k)=q^{*}$ exists with probability one. Hence, from Eq. (11), it can be seen that $q_{i}(k+1) \neq q_{i}(k)$ with a nonzero probability if and only if $q_{i}(k) \notin\{0,1\}$, and $\underline{q}(k+1)=\underline{q}(k)$ with probability one if and only if $q^{*} \in\{0,1\}$, where $\operatorname{Lim}_{k \rightarrow \infty} q_{i}(k)=q^{*}$, and hence the proof is completed.

Let $\Gamma_{i}(q)$ be the probability with which the algorithm 1 converges to the unit vector $e_{i}$ with initial probability vector $\underline{q}$ and defined as

$$
\Gamma_{i}(q)=\operatorname{prob}\left[q_{i}(\infty)=1 \mid \underline{q}(0)=\underline{q}\right]=\operatorname{prob}\left[q^{*}=e_{i} \mid \underline{q}(0)=\underline{q}\right]
$$

Let $C\left(S_{r}\right): S_{r} \rightarrow \Re$ be the state space of all real-valued continuously differentiable functions with bounded derivative defined on $S_{r}$, where $\Re$ is the real line. If $\psi(.) \in$ $C\left(S_{r}\right)$, the operator $U$ is defined by

$$
U \psi(q)=E[\psi(q(k+1) \mid q(k)=q]
$$

where $E[$.$] represents the mathematical expectation. It has been shown in Ref. 32$ that operator $U$ is linear and as the expectation of a nonnegative function remains nonnegative, operator $U$ preserves the non-negative functions. In other word, $U \psi(q) \geq 0$ for all $q \in S_{r}$, if $\psi(q) \geq 0$. If the operator $U$ is repeatedly applied $n$ times (for all $n>1$ ), we have

$$
U^{n-1} \psi(q)=E[\psi(q(k+1) \mid q(1)=q] .
$$

A function $\psi(q)$ is called super regular (sub regular) if and only if $\psi(q) \geq$ $U \psi(q)(\psi(q) \leq U \psi(q))$, for all $q \in S_{r}$. It has also been shown in Ref. 32 that $\Gamma_{i}(q)$ is the only continuous solution of $U \Gamma_{i}(q)=\Gamma_{i}(q)$, with the following boundary conditions.

$$
\begin{aligned}
& \Gamma_{i}\left(e_{i}\right)=1 \\
& \Gamma_{i}\left(e_{j}\right)=0 ; \quad j \neq i .
\end{aligned}
$$

Define

$$
\phi_{i}[x, q]=\frac{e^{-x q_{i} / a}-1}{e^{-x / a}-1}
$$

where $x>0$ is to be chosen. $\phi_{i}[x, q] \in C\left(S_{r}\right)$ and satisfies the boundary conditions above.

Theorem 2. Let $\psi_{i}(.) \in C\left(S_{r}\right)$ be super regular with $\psi_{i}\left(e_{i}\right)=1$ and $\psi_{i}\left(e_{j}\right)=0$ for $j \neq i$, then

$$
\psi_{i}(q) \geq \Gamma_{i}(q)
$$

for all $q \in S_{r}$. If $\psi_{i}(.) \in C\left(S_{r}\right)$ is sub regular with the same boundary conditions, then

$$
\psi_{i}(q) \leq \Gamma_{i}(q)
$$

for all $q \in S_{r}$. 
Proof. Theorem 2 has been proved in Ref. 32.

In what follows, we show that $\phi_{i}[x, q]$ is a sub regular function, thus $\phi_{i}[x, q]$ qualifies as a lower bound on $\Gamma_{i}(q)$. Since super and sub regular functions are closed under addition and multiplication by a positive constant, and if $\phi($.$) is super regular then$ $-\phi($.$) is sub regular, it follows that \phi_{i}[x, q]$ is sub regular if and only if

$$
\theta_{i}[x, q]=e^{\frac{-x q_{i}}{a}}
$$

is super regular. We now determine the conditions under which $\theta_{i}[x, q]$ is super regular. From the definition of operator $U$ given in Eq. (13), and Eq. (11) we have

$$
\begin{aligned}
& U \theta_{i}(x, q)=E\left[e^{-\frac{x q_{i}(k+1)}{a}} \mid q(k)=q\right] \\
& =\left[\sum_{j=1}^{r} q_{j} d_{j}^{*} e^{-\frac{x}{a}\left[\prod_{\substack{v_{m} \notin \omega_{j}, n=0 \\
o r \\
v_{m} \in \omega_{j}, n=1}}\left(p_{n}^{m}+a\left(1-p_{n}^{m}\right)\right)\right]} \quad+\sum_{j=1}^{r} q_{j} d_{j}^{*} e^{-\frac{x}{a}\left[\prod_{\substack{v_{m} \notin \omega_{j}, n=1 \\
o r \\
v_{m} \in \omega_{j}, n=0}}\left(p_{n}^{m}(1-a)\right)\right]}\right. \\
& =\left[q_{i} d_{i}^{*} e^{-\frac{x}{a}\left(q_{i}+a\left(1-q_{i}\right)\right)}+\sum_{j \neq i} q_{j} d_{j}^{*} e^{-\frac{x}{a}\left[\prod_{\substack{v_{m} \notin \omega_{j}, n=0 \\
o r \\
v_{m} \in \omega_{j}, n=1}}^{\prod_{n}}\left(p_{n}^{m}+a\left(1-p_{n}^{m}\right)\right)\right]}\right.
\end{aligned}
$$

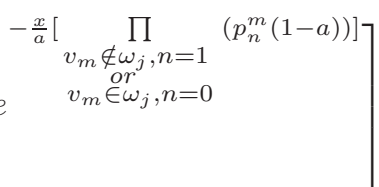

where $d_{j}{ }^{*}$ denotes the final value to which the reward probability $d_{j}(k)$ is converged, for large values of $k$, and

$$
e^{-\frac{x}{a}\left(q_{i}+a\left(1-q_{i}\right)\right)}
$$

is the expectation of $\theta_{i}(x, q)$, when the minimum WCDS $\omega_{i}$ is rewarded by the environment and it is calculated as the product of the rewarded probabilities of the dominator or dominatee vertices (the vertices which declare themselves as dominator and dominatees) which constructs WCDS $\omega_{i}$.

$$
\left.U \theta_{i}(x, q)=\left[q_{i} d_{i}^{*} e^{-\frac{x}{a}\left(q_{i}+a\left(1-q_{i}\right)\right)}+\sum_{j \neq i} q_{j} d_{j}^{*} e^{-\frac{x}{a}\left(q_{i}(1-a) \cdot \prod_{\substack{v_{m} \notin \omega_{j}, n=0 \\ o r \\ v_{m} \in \omega_{j}, n=1}} \frac{\left(p_{n}^{m}+a\left(1-p_{n}^{m}\right)\right)}{\left(p_{n}^{m}(1-a)\right)}\right.}\right)\right]
$$




$$
=\left[\sum_{j=i} q_{j} d_{j}^{*} e^{-\frac{x}{a} \rho_{j}^{i}\left(q_{i}+a\left(1-q_{i}\right)\right)}+\sum_{j \neq i} q_{j} d_{j}^{*} e^{-\frac{x}{a}\left(\rho_{j}^{i} q_{i}(1-a)\right)}\right]
$$

where $\rho_{j}^{i}>0$ and is defined as

$$
\begin{gathered}
\rho_{j}^{i}= \begin{cases}\prod_{v_{m} \notin \omega_{j}, n=0} \frac{\left(p_{n}^{m}+a\left(1-p_{n}^{m}\right)\right)}{\left(p_{n}^{m}(1-a)\right)} ; & i \neq j \\
v_{m} \in \omega_{j}, n=1 & i=j \text { or } \quad\left(\omega_{i} \cap \omega_{j}\right)=\phi \\
1 ; & \end{cases} \\
U \theta_{i}(x, q)-\theta_{i}(x, q)=\left[e^{-\frac{x q_{i} \rho_{j}^{i}}{a}} \sum_{j=i} q_{j} d_{j}^{*} e^{-x\left(1-q_{i}\right) \rho_{j}^{i}}+e^{-\frac{x q_{i} \rho_{j}^{i}}{a}} \sum_{j \neq i} q_{j} d_{j}^{*} e^{x q_{i} \rho_{j}^{i}}\right]-e^{-\frac{x q_{i}}{a}}
\end{gathered}
$$

$\theta_{i}(x, q)$ is super regular if

$$
e^{-\frac{x q_{i} \rho_{j}^{i}}{a}} \sum_{j=i} q_{j} d_{j}^{*} e^{-x\left(1-q_{i}\right) \rho_{j}^{i}}+e^{-\frac{x q_{i} \rho_{j}^{i}}{a}} \sum_{j \neq i} q_{j} d_{j}^{*} e^{x q_{i} \rho_{j}^{i}} \leq e^{-\frac{x q_{i}}{a}}
$$

and

$$
U \theta_{i}(x, q) \leq e^{-\frac{x q_{i}}{a}} q_{i} d_{i}^{*} e^{-x\left(1-q_{i}\right)}+e^{-\frac{x q_{i}}{a}} \sum_{j \neq i} q_{j} d_{j}^{*} e^{x q_{i}}
$$

if $\theta_{i}(x, q)$ is super regular, so we have

$$
U \theta_{i}(x, q)-\theta_{i}(x, q) \leq\left[e^{-\frac{x q_{i}}{a}} q_{i} d_{i}^{*} e^{-x\left(1-q_{i}\right)}+e^{-\frac{x q_{i}}{a}} \sum_{j \neq i} q_{j} d_{j}^{*} e^{x q_{i}}\right]-e^{-\frac{x q_{i}}{a}} .
$$

After multiplying and dividing the right hand side of the inequality above by $-x q_{i}$ and some algebraic simplifications, we have

$$
\begin{aligned}
U \theta_{i}(x, q)-\theta_{i}(x, q) & \leq-x q_{i} e^{\frac{-x q_{i}}{a}}\left[q_{i} d_{i}^{*} \frac{e^{-x\left(1-q_{i}\right)}-1}{-x q_{i}}-\sum_{j \neq i} q_{j} d_{j}^{*} \frac{e^{x q_{i}}-1}{x q_{i}}\right] \\
& =-x q_{i} e^{\frac{-x q_{i}}{a}}\left[d_{i}^{*} \frac{e^{-x\left(1-q_{i}\right)}-1}{-x}-\sum_{j \neq i} q_{j} d_{j}^{*} \frac{e^{x q_{i}}-1}{x q_{i}}\right] \\
& =-x q_{i} e^{\frac{-x q_{i}}{a}}\left[\left(1-q_{i}\right) d_{i}^{*} \frac{e^{-x\left(1-q_{i}\right)}-1}{-x\left(1-q_{i}\right)}-\sum_{j \neq i} q_{j} d_{j}^{*} \frac{e^{x q_{i}}-1}{x q_{i}}\right]
\end{aligned}
$$

and

$$
V[u]= \begin{cases}\frac{e^{u}-1}{u} ; & u \neq 0 \\ 1 ; & u=0\end{cases}
$$




$$
\begin{aligned}
U \theta_{i}(x, q)-\theta_{i}(x, q) & \leq-x q_{i} e^{\frac{-x q_{i}}{a}}\left[(1-q)_{i} d_{i}^{*} V\left[-x\left(1-q_{i}\right)\right]-\left(\sum_{j \neq i} q_{j} d_{j}^{*}\right) V\left[x q_{i}\right]\right] \\
& =-x q_{i} \theta_{i}(x, q) G_{i}(x, q)
\end{aligned}
$$

where $G_{i}(x, q)$ is defined as

$$
G_{i}(x, q)=\left(1-q_{i}\right) d_{i}^{*} V\left[-x\left(1-q_{i}\right)\right]-\left(\sum_{j \neq i} q_{j} d_{j}^{*}\right) V\left[x q_{i}\right] .
$$

Therefore, $\theta_{i}(x, q)$ is super regular if

$$
G_{i}(x, q) \geq 0
$$

for all $q \in S_{r}$. From Eq. (16), it follows that $\theta_{i}(x, q)$ is super regular if we have

$$
f_{i}(x, q)=\frac{V\left[-x\left(1-q_{i}\right)\right]}{V\left[x q_{i}\right]} \leq \frac{\sum_{j \neq i} q_{j} d_{j}^{*}}{\left(1-q_{i}\right) d_{i}^{*}} .
$$

The right hand side of the inequality (18) consists of the non-negative terms, so we have

$$
\left(\sum_{j \neq i} q_{j}\right) \min _{j \neq i}\left(\frac{d_{j}^{*}}{d_{i}^{*}}\right) \leq \frac{1}{\left(1-q_{i}\right)} \sum_{j \neq i} q_{j} \frac{d_{j}^{*}}{d_{i}^{*}} \leq\left(\sum_{j \neq i} q_{j}\right) \max _{j \neq i}\left(\frac{d_{j}^{*}}{d_{i}^{*}}\right) .
$$

Substituting $\sum_{j \neq i} q_{j}$ by $\left(1-q_{i}\right)$ in the above inequality, we can rewrite it as

$$
\min _{j \neq i}\left(\frac{d_{j}^{*}}{d_{i}^{*}}\right) \leq \frac{\sum_{j \neq i} q_{j} \frac{d_{j}^{*}}{d_{i}^{*}}}{\sum_{j \neq i} q_{j}} \leq \max _{j \neq i}\left(\frac{d_{j}^{*}}{d_{i}^{*}}\right) .
$$

From Eq. (18), it follows that $\theta_{i}(x, q)$ is super regular if we have

$$
f_{i}(x, q) \geq \max _{j \neq i}\left(d_{j}^{*} / d_{i}^{*}\right) .
$$

For further simplification, let employ logarithms. Let

$$
\Delta(q, x)=\ln f_{i}(x, q) .
$$

It has also been shown in Ref. 36 that

$$
\begin{gathered}
-\int_{0}^{x} H^{\prime}(u) d u \leq \Delta(q, x) \leq-\int_{-x}^{0} H^{\prime}(u) d u \\
H(u)=\frac{d H(u)}{d u}, \quad H(u)=\ln V(u) .
\end{gathered}
$$

We have

$$
\frac{1}{V[x]} \leq \frac{V\left[-x\left(1-q_{i}\right)\right]}{V\left[x q_{i}\right]} \leq V[-x]
$$

and

$$
\frac{1}{V[x]}=\max _{j \neq i}\left(\frac{d_{j}^{*}}{d_{i}^{*}}\right) .
$$


Let $x^{*}$ be the value of $x$ for which the Eq. (19) is true. It is shown that, there exists a value of $x>0$ under which the Eq. (19) is satisfied, if $\left(d_{j} / d_{i}\right)$ is smaller than 1 for all $j \neq i$. By choosing a value $x=x^{*}$, the Eq. (19) holds. Consequently Eq. (17) is true and $\theta_{i}(x, q)$ is a super regular function, thus

$$
\phi_{i}[x, q]=\frac{e^{-x q_{i} / a-1}}{e^{-x / a}-1}
$$

is a sub regular function satisfying the boundary conditions (14) and from Theorem 2 and inequality (15), we conclude that

$$
\phi_{i}[x, q] \leq \Gamma_{i}(q) \leq 1 .
$$

From the definition of $\phi_{i}[x, q]$, we see that given any $\varepsilon>0$ there exists a positive constant $a^{*}<1$ such that for all $0<a \leq a^{*}$, we have

$$
1-\varepsilon \leq \phi_{i}[x, q] \leq \Gamma_{i}(q) \leq 1 .
$$

Thus we conclude that the probability with which the algorithm 1 converges to (or constructs) the WCDS with the minimum expected weight is equal to 1 as $k \rightarrow \infty$, and this completes the proof of the Theorem 1 .

Theorem 3. Let $q_{i}(k)$ be the probability of constructing the minimum $W C D S \omega_{i}$, at stage $k$, and $(1-\varepsilon)$ be the probability with which algorithm 1 converges to the $W C D S \omega_{i}$. If $\underline{q}(k)$ is updated by algorithm 1 , then for every error parameter $\varepsilon \in$ $(0,1)$, there exists a learning rate $a \in(\varepsilon, q)$ so that,

$$
\frac{x a}{e^{x a}-1}=\max _{j \neq i}\left(\frac{d_{j}}{d_{i}}\right)
$$

where $1-e^{-x q_{i}}=\left(1-e^{-x}\right) \cdot(1-\varepsilon)$ and $q_{i}=\left[q_{i}(k) \mid k=0\right]$.

Proof. It has been proved in Refs. 32 and 36 that there always exists a $x>0$ under which the Eq. (19) is satisfied, if $d_{j} / d_{i}<1$, for all $j \neq i$. Hence, it is concluded that

$$
\phi_{i}[x, q] \leq \Gamma_{i}(q) \leq \frac{1-e^{-x q_{i}}}{1-e^{-x}}
$$

where $q_{i}$ is the initial probability of the optimal WCDS $\omega_{i}$. It is assumed in Theorem 1 that the probability with which the algorithm 1 converges to the WCDS with the minimum expected weight is $(1-\varepsilon)$, for each $0<a<a^{*}$, where $a^{*}(\varepsilon) \in(0,1)$. So we conclude that,

$$
\frac{1-e^{-x q_{i}}}{1-e^{-x}}=1-\varepsilon
$$

It can be shown that ${ }^{36,48}$ for every error parameter $\varepsilon \in(0,1)$ there exists a value of $x$ under which the Eq. (19) is satisfied, and we have

$$
\frac{x^{*} a}{e^{x * a}-1}=\max _{j \neq i}\left(\frac{d_{j}^{*}}{d_{i}^{*}}\right) \text {. }
$$


It is concluded that for every error parameter $\varepsilon \in(0,1)$ there exists a learning rate $a \in(\varepsilon, q)$ under which the probability with which the algorithm 1 is converged to the WCDS with the minimum expected weight is greater than $(1-\varepsilon)$ and hence the proof of the theorem.

\section{Experimental Results}

To study the performance of the proposed algorithms, we have conducted two groups of simulation. The first group is concerned with investigating the efficiency of the proposed algorithms on three stochastic graphs borrowed from Ref. 49 and shown in Figs. 8, 9, and 10, and the second group of the simulation evaluates the scalability of the proposed algorithms on dense stochastic graphs. In all the experiments presented in this paper, the number of samples taken by the proposed algorithms from the stochastic graph to construct the WCDS with the minimum expected weight is compared with that of the standard sampling method quoted in Appendix A. The reinforcement scheme used for updating the action probability

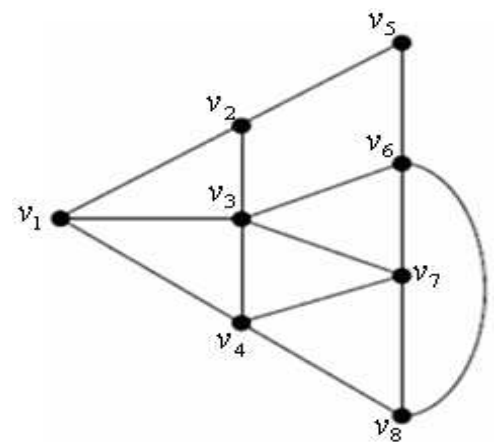

\begin{tabular}{ccc}
\hline Vertex & Weight & Probability \\
\hline $\mathrm{v}_{1}$ & $\{2,8,12\}$ & $\{0.9,0.08,0.02\}$ \\
$\mathrm{v}_{2}$ & $\{10,24,35\}$ & $\{0.85,0.12,0.03\}$ \\
$\mathrm{v}_{3}$ & $\{6,18,24\}$ & $\{0.88,0.1,0.02\}$ \\
$\mathrm{v}_{4}$ & $\{12,22,30\}$ & $\{0.85,0.11,0.04\}$ \\
$\mathrm{v}_{5}$ & $\{17,35,50\}$ & $\{0.75,0.2,0.05\}$ \\
$\mathrm{v}_{6}$ & $\{3,7,10\}$ & $\{0.68,0.25,0.07\}$ \\
$\mathrm{v}_{7}$ & $\{4,19,15\}$ & $\{0.75,0.14,0.11\}$ \\
$\mathrm{v}_{8}$ & $\{5,10,12\}$ & $\{0.65,0.23,0.12\}$ \\
\hline
\end{tabular}

Fig. 8. Stochastic Graph 1 and its probability distribution function.

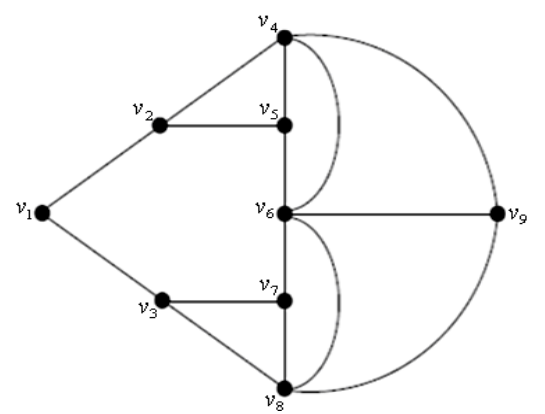

\begin{tabular}{ccc}
\hline Vertex & Weight & Probability \\
\hline $\mathrm{V}_{1}$ & $\{2,8,12\}$ & $\{0.9,0.08,0.02\}$ \\
$\mathrm{V}_{2}$ & $\{10,24,35\}$ & $\{0.85,0.12,0.03\}$ \\
$\mathrm{V}_{3}$ & $\{6,18,24\}$ & $\{0.88,0.1,0.02\}$ \\
$\mathrm{V}_{4}$ & $\{12,22,30\}$ & $\{0.85,0.11,0.04\}$ \\
$\mathrm{V}_{5}$ & $\{17,35,50\}$ & $\{0.75,0.2,0.05\}$ \\
$\mathrm{V}_{6}$ & $\{3,7,10\}$ & $\{0.68,0.25,0.07\}$ \\
$\mathrm{V}_{7}$ & $\{4,19,15\}$ & $\{0.75,0.14,0.11\}$ \\
$\mathrm{V}_{8}$ & $\{5,10,12\}$ & $\{0.65,0.23,0.12\}$ \\
$\mathrm{V}_{9}$ & $\{10,19,24\}$ & $\{0.80,0.14,0.06\}$ \\
\hline
\end{tabular}

Fig. 9. Stochastic Graph 2 and its probability distribution function. 


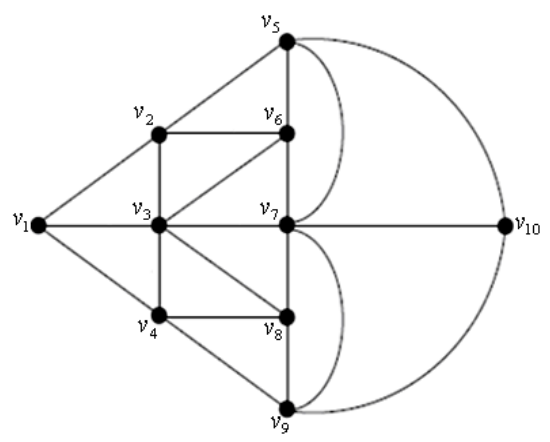

\begin{tabular}{ccc}
\hline Vertex & Weight & Probability \\
\hline $\mathrm{v}_{1}$ & $\{2,8,12\}$ & $\{0.9,0.08,0.02\}$ \\
$\mathrm{v}_{2}$ & $\{10,24,35\}$ & $\{0.85,0.12,0.03\}$ \\
$\mathrm{v}_{3}$ & $\{6,18,24\}$ & $\{0.88,0.1,0.02\}$ \\
$\mathrm{v}_{4}$ & $\{12,22,30\}$ & $\{0.85,0.11,0.04\}$ \\
$\mathrm{v}_{5}$ & $\{17,35,50\}$ & $\{0.75,0.2,0.05\}$ \\
$\mathrm{v}_{6}$ & $\{3,7,10\}$ & $\{0.68,0.25,0.07\}$ \\
$\mathrm{v}_{7}$ & $\{4,19,15\}$ & $\{0.75,0.14,0.11\}$ \\
$\mathrm{V}_{8}$ & $\{5,10,12\}$ & $\{0.65,0.23,0.12\}$ \\
$\mathrm{v}_{9}$ & $\{10,19,24\}$ & $\{0.80,0.14,0.06\}$ \\
$\mathrm{v}_{10}$ & $\{18,27,36\}$ & $\{0.94,0.05,0.01\}$ \\
\hline
\end{tabular}

Fig. 10. Stochastic Graph 3 and its probability distribution function.

vectors is $L_{R-I}$, and each algorithm is terminated when the probability of the constructed WCDS (PWCDS) is 0.95 or greater, or the number of constructed WCDSs exceeds a pre-defined threshold.

In the first group of the simulations, the proposed algorithms are tested on Graphs 1, 2, and 3, and the results are summarized in Tables 2, 4, and 6 for the different values of learning rate $a$ which varies from 0.004 to 0.100 . Then, the obtained results in terms of the number of samples taken from the graph are compared with those of the standard sampling method given in Tables 1, 3, and 5 . The aim of the standard sampling method used in our experiments is to obtain the minimum number of samples needs to be taken from the stochastic graph such that the average weight of the samples taken from the optimal WCDS be in the interval $(\mu-\delta, \mu+\delta)$ with probability $1-\varepsilon$, where $\varepsilon$ is the error parameter and $\delta$ is a small enough positive number. Graph 1 which is shown in Fig. 8 has 8 vertices, 12 edges and its minimal WCDS is $\delta_{1}{ }^{*}=\left\{v_{1}, v_{6}\right\}$, Graph 2 which is shown in Fig. 9 has 9 vertices, 15 edges and its minimal WCDS is $\delta_{2}{ }^{*}=\left\{v_{1}, v_{6}\right\}$, and Graph 3 which is shown in Fig. 10 has 10 vertices, 21 edges and its minimal WCDS is $\delta_{3}{ }^{*}=\left\{v_{1}, v_{7}\right\}$. The probability distributions of the vertex weight of these three stochastic graphs are given in tables shown in Figs. 8, 9, and 10. The simulation results given in Tables 2, 4, and 6 are averaged over 1000 runs and each of these tables includes the information about the total number of samples taken from all vertices of the graph (TS), the total number of the samples taken from the vertices of the WCDS with the minimum expected weight (WCDS) and the percentage of the converged runs (the percentage of runs converged to the minimum WCDS) (PC).

According to the standard sampling method described in appendix A, to obtain a confidence level $1-\varepsilon$ for the WCDS, we need to build a confidence with level $1-\varepsilon_{i}$ for each vertex $v_{i}$ such that $\sum_{i=1}^{k} \varepsilon_{i}=\varepsilon$. We assume that the vertices of the stochastic graph all have the same confidence level $1-\varepsilon_{0}$. Therefore, selecting $\varepsilon_{0}=\varepsilon / k$, where $k$ denotes the cardinality of the WCDS, guarantees a confidence level $1-\varepsilon$ for the WCDS. For instance, when a confidence level $80 \%$ is desired for the WCDS of Graph 1, we need to build a confidence with level $90 \%$ for each of the 
Table 1. The total number of samples taken from Graph 1 in standard sampling method.

\begin{tabular}{crrrrrrrrrrr}
\hline Vertex & \multicolumn{10}{c}{ Confidence Level for WCDS } \\
\cline { 2 - 12 } & 0.5 & 0.6 & 0.7 & 0.75 & 0.8 & 0.85 & 0.9 & 0.925 & 0.95 & 0.975 & 0.99 \\
\hline v1 & 317 & 286 & 259 & 308 & 243 & 282 & 269 & 346 & 310 & 448 & 369 \\
v2 & 521 & 518 & 541 & 466 & 474 & 468 & 501 & 517 & 608 & 454 & 450 \\
v3 & 353 & 337 & 373 & 333 & 381 & 354 & 353 & 421 & 415 & 492 & 465 \\
v4 & 406 & 395 & 345 & 377 & 418 & 309 & 448 & 309 & 436 & 391 & 491 \\
v5 & 590 & 697 & 642 & 685 & 630 & 808 & 753 & 631 & 610 & 729 & 699 \\
v6 & 340 & 265 & 304 & 273 & 314 & 241 & 315 & 356 & 336 & 371 & 376 \\
v7 & 311 & 320 & 277 & 312 & 315 & 328 & 377 & 330 & 354 & 392 & 339 \\
v8 & 333 & 369 & 377 & 395 & 377 & 471 & 388 & 423 & 376 & 387 & 439 \\
\hline Total & 3171 & 3187 & 3118 & 3149 & 3152 & 3261 & 3404 & 3333 & 3445 & 3664 & 3628 \\
\hline
\end{tabular}

8 vertices. ${ }^{50}$ The results of the standard sampling method for Graphs 1, 2, and 3 to obtain a confidence level $1-\varepsilon$ for the WCDS, as $\varepsilon$ varies from 0.01 to 0.5 have been shown in Tables 1, 2, and 3, respectively.

From the results of the simulations, the following points can be made.

The experiments have shown that for all algorithms the total number of converged runs (convergence rate) increases as the learning rate decreases. For example, in algorithm 1 for Graph 1, the number of converged runs is $54.3 \%$, when $\alpha=0.07$, whereas it is $98.5 \%$ when $\alpha=0.007$. (see Table 2)

The simulation results show that the total number of samples taken by algorithm 2 from the vertices of the graph is less than those of algorithm 1, but the percentage of the runs converged to the optimal WCDS is higher. The reason for this reduction in number of samples taken by algorithm 2 from the graph is the fact that, when a vertex declares itself as a dominator and its weight is sampled, unlike algorithm 1, algorithm 2 does not need to sample the weight of its neighbors again. This sampling method results in decreasing unnecessary samples and hence decreasing the running time of algorithm 2. The results show that for the same convergence rate, algorithm 2 has lower sampling rate comparing algorithm 1 .

Experiments show that algorithm 3 has a lower convergence rate comparing algorithm 2. This may be due to the fact that the action set of each learning automaton in algorithm 3 is significantly larger than that of in algorithm 2, specifically for the dense graphs. That is, in algorithm 3 as stated in Sec. 3, each learning automaton has many choices (the number of actions available for each automaton is equal to the number of its neighbors plus one), while in algorithm 2 each learning automaton has at most two possible actions. This results in prolonging the convergence of each learning automaton to its optimal action and so the proposed algorithm to the optimal WCDS.

Comparing the results given in Tables 2, 4, and 6 for algorithms 1 and 3, we observe that the total number of samples taken by algorithm 3 is not much more than that of algorithm 1, while it is expected to be due to the larger number of actions in algorithm 3 . The reason for this reduction in number of samples taken by 
Table 2. The average number of samples taken from the graph and the minimum WCDS, and the percentage of the converged runs for Graph 1.

\begin{tabular}{|c|c|c|c|c|c|c|c|c|c|c|c|c|c|c|c|}
\hline \multirow{2}{*}{$\begin{array}{l}\text { Learning } \\
\text { rate }\end{array}$} & \multicolumn{3}{|c|}{ Algorithm 1} & \multicolumn{3}{|c|}{ Algorithm 2} & \multicolumn{3}{|c|}{ Algorithm 3} & \multicolumn{3}{|c|}{ Algorithm 4} & \multicolumn{3}{|c|}{ Algorithm 5} \\
\hline & TS & WCDS & $\mathrm{PC}$ & TS & WCDS & $\mathrm{PC}$ & TS & WCDS & $\mathrm{PC}$ & $\mathrm{TS}$ & WCDS & $\mathrm{PC}$ & TS & WCDS & $\mathrm{PC}$ \\
\hline 0.004 & 4909.7 & 1675.4 & 100.0 & 2719.3 & 1994.3 & 100.0 & 13732.3 & 7163.4 & 100.0 & 2433.1 & 1035.2 & 100.0 & 3750.8 & 1454.8 & 100.0 \\
\hline 0.006 & 3332.0 & 1129.5 & 100.0 & 1947.5 & 1424.4 & 100.0 & 9468.3 & 5506.4 & 100.0 & 1647.9 & 692.8 & 100.0 & 2499.4 & 1091.2 & 99.0 \\
\hline 0.007 & 2844.6 & 963.5 & 98.5 & 1707.1 & 1248.6 & 100.0 & 4762.8 & 1305.1 & 100.0 & 1412.8 & 594.4 & 100.0 & 2277.4 & 1060.1 & 97.0 \\
\hline 0.008 & 2495.3 & 843.6 & 98.2 & 1517.1 & 1110.5 & 100.0 & 4499.3 & 1359.0 & 100.0 & 1237.0 & 518.9 & 100.0 & 2089.7 & 1058.3 & 99.0 \\
\hline 0.02 & 1015.8 & 331.3 & 87.5 & 456.6 & 331.6 & 100.0 & 2127.5 & 1356.5 & 47.4 & 553.2 & 224.7 & 96.9 & 1000.1 & 589.3 & 93.8 \\
\hline 0.03 & 673.5 & 213.3 & 81.6 & 346.4 & 250.5 & 100.0 & 1283.5 & 730.9 & 32.0 & 395.0 & 173.6 & 91.9 & 588.6 & 326.0 & 90.8 \\
\hline 0.04 & 496.8 & 155.4 & 73.0 & 276.2 & 197.0 & 100.0 & 1341.8 & 800.0 & 33.7 & 292.3 & 131.8 & 90.4 & 431.8 & 250.3 & 82.4 \\
\hline 0.05 & 386.8 & 119.1 & 70.1 & 228.1 & 162.8 & 100.0 & 943.5 & 532.4 & 22.1 & 229.2 & 104.8 & 87.9 & 279.5 & 141.1 & 77.2 \\
\hline 0.06 & 314.3 & 95.5 & 59.4 & 198.4 & 139.2 & 100.0 & 677.4 & 368.4 & 14.6 & 182.8 & 82.9 & 82.1 & 234.6 & 117.0 & 76.4 \\
\hline 0.07 & 268.9 & 83.3 & 54.3 & 196.0 & 138.7 & 100.0 & 523.9 & 269.2 & 13.1 & 152.1 & 70.0 & 79.8 & 204.2 & 109.9 & 75.2 \\
\hline
\end{tabular}


Table 3. The total number of samples taken from Graph 2 in standard sampling method.

\begin{tabular}{crrrrrrrrrrr}
\hline Vertex & \multicolumn{10}{c}{ Confidence Level for WCDS } \\
\cline { 2 - 12 } & 0.5 & 0.6 & 0.7 & 0.75 & 0.8 & 0.85 & 0.9 & 0.925 & 0.95 & 0.975 & 0.99 \\
\hline v1 & 317 & 286 & 259 & 308 & 243 & 282 & 269 & 346 & 310 & 448 & 369 \\
v2 & 521 & 518 & 541 & 466 & 474 & 468 & 501 & 517 & 608 & 454 & 450 \\
v3 & 353 & 337 & 373 & 333 & 381 & 354 & 353 & 421 & 415 & 492 & 465 \\
v4 & 406 & 395 & 345 & 377 & 418 & 309 & 448 & 309 & 436 & 391 & 491 \\
v5 & 590 & 697 & 642 & 685 & 630 & 808 & 753 & 631 & 610 & 729 & 699 \\
v6 & 340 & 265 & 304 & 273 & 314 & 241 & 315 & 356 & 336 & 371 & 376 \\
v7 & 311 & 320 & 277 & 312 & 315 & 328 & 377 & 330 & 354 & 392 & 339 \\
v8 & 333 & 369 & 377 & 395 & 377 & 471 & 388 & 423 & 376 & 387 & 439 \\
v9 & 242 & 250 & 300 & 343 & 312 & 371 & 339 & 294 & 328 & 363 & 433 \\
\hline Total & 3413 & 3437 & 3418 & 3492 & 3464 & 3632 & 3743 & 3627 & 3773 & 4027 & 4061 \\
\hline
\end{tabular}

algorithm 3 can be the fact that algorithm 3 guarantees formation of a WCDS at each stage, while in a number of stages algorithm 1 fails in constructing a WCDS for the stochastic graph, which results in increasing the number of samples needs to be taken by the algorithm.

The simulation results show that algorithm 4 greatly improves the convergence rate. The reason for higher convergence rate for this algorithm is the fact that it employs the variable action set learning automata to construct the WCDSs which are more likely to be the WCDS with the minimum expected weight, and so increases the convergence rate to the optimal WCDS. The results show that algorithm 4 also considerably outperforms algorithms $1,2,3$, and 5 in terms of the sampling rate.

According to the results given in Tables 2, 4, and 6, algorithm 5 has higher convergence rate than both algorithms 1 and 3 . The number of samples needs to be taken by this algorithm to construct the minimum WCDS is also much less than that of algorithms 1 and 3 .

Comparing the results given in Tables 1,3 , and 5 with Tables 2 , 4, and 6 , we find that the number of samples needs to be taken by the proposed algorithms, when the algorithms converge to the minimum WCDS with probability $1-\varepsilon$, is considerably less than that of the standard sampling method. This study is the main result of the paper that was theoretically proved in the previous section and will be considered in more details at the end of this section.

Another property we want to illustrate is any time behavior of the proposed algorithms. We show this property by plotting the development of the probability of choosing the minimum WCDS in time. Figures 11, 12 and 13 show a rapid progress in the beginning and flattening out later on. This is typical for many algorithms that work by iterative improvements on the initial solution. Based on the any time curve depicted in Figs. 11, 12 and 13 we can make some general observation concerning termination condition for the proposed algorithms. We can divide the run time into two equally long sections, the first and the second half. As Figs. 11, 12 and 13 indicate, the progress in terms of increments in probability of choosing the 
Table 4. The average number of samples taken from the graph and the minimum WCDS, and the percentage of the converged runs for Graph 2.

\begin{tabular}{|c|c|c|c|c|c|c|c|c|c|c|c|c|c|c|c|}
\hline $\begin{array}{c}\text { Learning } \\
\text { rate }\end{array}$ & \multicolumn{3}{|c|}{ Algorithm 1} & \multicolumn{3}{|c|}{ Algorithm 2} & \multicolumn{3}{|c|}{ Algorithm 3} & \multicolumn{3}{|c|}{ Algorithm 4} & \multicolumn{3}{|c|}{ Algorithm 5} \\
\hline 0.005 & 3794.3 & 1001.7 & 100.0 & 3699.5 & 2231.2 & 100.0 & 5585.8 & 839.4 & 100.0 & 3080.7 & 869.2 & 100.0 & 2721.2 & 1106.1 & 100.0 \\
\hline 0.006 & 3183.4 & 836.9 & 99.9 & 3043.8 & 1861.7 & 100.0 & 4759.9 & 702.6 & 100.0 & 2536.6 & 716.2 & 100.0 & 2146.7 & 702.1 & 100.0 \\
\hline 0.008 & 2412.5 & 622.9 & 99.4 & 2322.0 & 1395.4 & 100.0 & 3723.0 & 527.9 & 100.0 & 1964.5 & 535.7 & 98.0 & 1733.8 & 619.4 & 100.0 \\
\hline 0.009 & 2151.5 & 553.4 & 99.3 & 2040.6 & 1244.2 & 100.0 & 3389.0 & 468.9 & 100.0 & 1744.1 & 558.6 & 96.0 & 1632.6 & 632.7 & 99.0 \\
\hline 0.01 & 1928.6 & 498.0 & 99.1 & 1840.2 & 1113.5 & 99.1 & 3048.8 & 421.6 & 99.6 & 1725.3 & 570.6 & 94.0 & 1433.7 & 487.5 & 99.0 \\
\hline 0.02 & 983.4 & 241.6 & 94.8 & 926.1 & 557.5 & 96.0 & 2241.9 & 825.6 & 78.6 & 1619.8 & 499.5 & 93.0 & 836.3 & 377.0 & 91.0 \\
\hline 0.05 & 392.9 & 94.6 & 75.4 & 371.3 & 222.7 & 86.6 & 1074.0 & 633.4 & 24.8 & 371.2 & 206.6 & 56.5 & 297.6 & 128.2 & 83.2 \\
\hline 0.06 & 323.9 & 76.8 & 66.6 & 303.2 & 184.0 & 84.9 & 850.3 & 516.4 & 12.8 & 354.9 & 186.6 & 56.0 & 220.2 & 91.0 & 75.4 \\
\hline 0.07 & 268.2 & 65.7 & 66.1 & 261.7 & 158.6 & 85.0 & 590.0 & 315.4 & 13.8 & 211.7 & 107.8 & 53.9 & 186.3 & 78.6 & 78.8 \\
\hline 0.08 & 236.3 & 61.0 & 51.6 & 224.0 & 136.9 & 82.8 & 478.7 & 246.6 & 6.2 & 175.8 & 88.2 & 55.0 & 157.9 & 70.8 & 75.6 \\
\hline 0.09 & 212.9 & 53.1 & 52.5 & 199.1 & 121.4 & 82.3 & 419.0 & 220.9 & 5.8 & 148.9 & 75.1 & 51.2 & 137.6 & 60.2 & 74.2 \\
\hline 0.1 & 190.3 & 53.6 & 45.5 & 176.9 & 109.3 & 80.6 & 353.3 & 190.5 & 4.8 & 129.6 & 64.2 & 48.3 & 132.1 & 57.7 & 73.4 \\
\hline
\end{tabular}


Table 5. The total number of samples taken from Graph 3 in standard sampling method.

\begin{tabular}{lrrrrrrrrrrr}
\hline Vertex & \multicolumn{10}{c}{ Confidence Level for WCDS } \\
\cline { 2 - 12 } & 0.5 & 0.6 & 0.7 & 0.75 & 0.8 & 0.85 & 0.9 & 0.925 & 0.95 & 0.975 & 0.99 \\
\hline v1 & 317 & 286 & 259 & 308 & 243 & 282 & 269 & 346 & 310 & 448 & 369 \\
v2 & 521 & 518 & 541 & 466 & 474 & 468 & 501 & 517 & 608 & 454 & 450 \\
v3 & 353 & 337 & 373 & 333 & 381 & 354 & 353 & 421 & 415 & 492 & 465 \\
v4 & 406 & 395 & 345 & 377 & 418 & 309 & 448 & 309 & 436 & 391 & 491 \\
v5 & 590 & 697 & 642 & 685 & 630 & 808 & 753 & 631 & 610 & 729 & 699 \\
v6 & 340 & 265 & 304 & 273 & 314 & 241 & 315 & 356 & 336 & 371 & 376 \\
v7 & 311 & 320 & 277 & 312 & 315 & 328 & 377 & 330 & 354 & 392 & 339 \\
v8 & 333 & 369 & 377 & 395 & 377 & 471 & 388 & 423 & 376 & 387 & 439 \\
v9 & 242 & 250 & 300 & 343 & 312 & 371 & 339 & 294 & 328 & 363 & 433 \\
v10 & 386 & 425 & 465 & 480 & 485 & 446 & 454 & 464 & 437 & 439 & 502 \\
\hline Total & 3802 & 3867 & 3887 & 3977 & 3953 & 4084 & 4201 & 4096 & 4214 & 4469 & 4568 \\
\hline
\end{tabular}

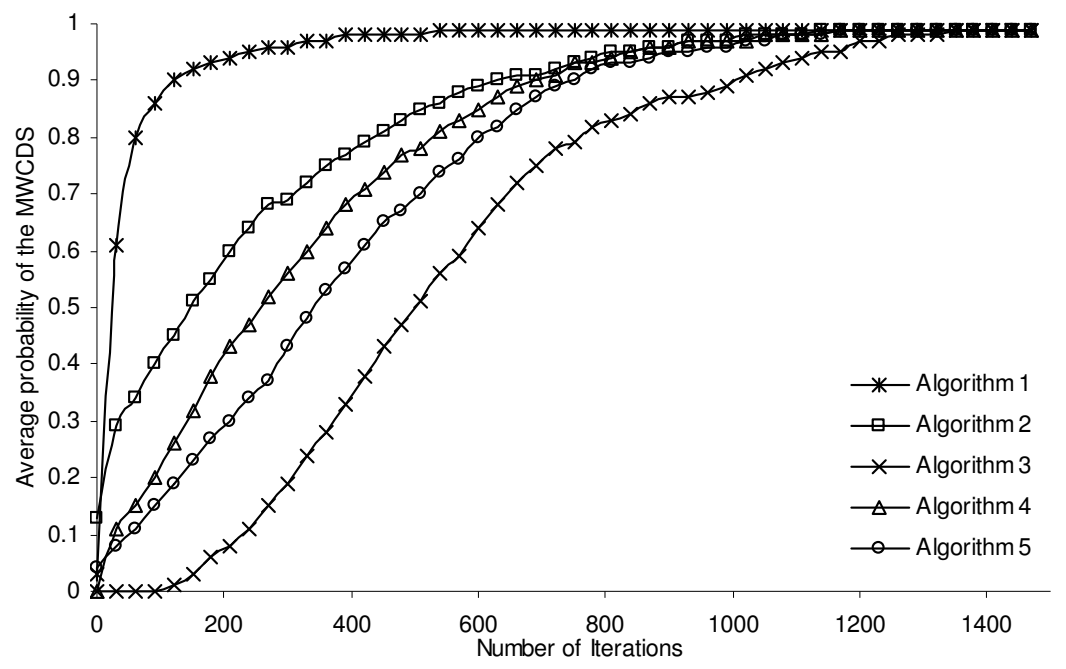

Fig. 11. The probability of the minimum WCDS for Graph 1.

minimum WCDS is significantly greater than the achievements in the second half. This provides a general suggestion that it might not be worthwhile to allow very long runs; because of the any time behavior of the proposed algorithms efforts spent after a certain time may not result in better solution quality. A given point in any time curve gives us the average number of iterations required to find the minimum WCDS with a certain probability. This is one advantage of the proposed algorithm over non-iterative algorithms. Probability of the minimum WCDS (PWCDS) at each instant of the proposed algorithms is defined as the product of the probability of choosing the vertices of the constructed WCDS. The plots of the average probability 
Table 6. The average number of samples taken from the graph and the minimum WCDS, and the percentage of the converged runs for Graph 3.

\begin{tabular}{|c|c|c|c|c|c|c|c|c|c|c|c|c|c|c|c|}
\hline \multirow{2}{*}{$\begin{array}{l}\text { Learning } \\
\text { rate }\end{array}$} & \multicolumn{3}{|c|}{ Algorithm 1} & \multicolumn{3}{|c|}{ Algorithm 2} & \multicolumn{3}{|c|}{ Algorithm 3} & \multicolumn{3}{|c|}{ Algorithm 4} & \multicolumn{3}{|c|}{ Algorithm 5} \\
\hline & TS & WCDS & $\mathrm{PC}$ & TS & WCDS & $\mathrm{PC}$ & TS & WCDS & $\mathrm{PC}$ & TS & WCDS & $\mathrm{PC}$ & TS & WCDS & $\mathrm{PC}$ \\
\hline 0.004 & 5561.5 & 1543.4 & 100.0 & 4301.1 & 2699.1 & 100.0 & 15981.1 & 7422.8 & 100.0 & 2826.1 & 1056.4 & 100.0 & 5098.2 & 2892.7 & 100.0 \\
\hline 0.006 & 3779.9 & 1045.6 & 96.6 & 2880.9 & 1801.8 & 100.0 & 7549.3 & 1922.4 & 100.0 & 1913.1 & 702.2 & 100.0 & 2989.1 & 1207.4 & 100.0 \\
\hline 0.007 & 3262.4 & 902.4 & 93.9 & 2468.4 & 1542.9 & 100.0 & 6697.9 & 1660.3 & 100.0 & 1656.4 & 602.4 & 100.0 & 2659.4 & 1131.2 & 100.0 \\
\hline 0.008 & 2843.0 & 777.5 & 93.0 & 2164.2 & 1352.2 & 100.0 & 5919.9 & 1561.6 & 100.0 & 1451.2 & 529.3 & 100.0 & 2338.4 & 1046.5 & 98.6 \\
\hline 0.02 & 1131.0 & 296.4 & 82.1 & 871.6 & 539.5 & 99.5 & 3918.5 & 2294.2 & 36.8 & 652.4 & 244.3 & 97.0 & 1398.3 & 943.1 & 91.0 \\
\hline 0.03 & 750.1 & 187.2 & 71.7 & 587.3 & 360.4 & 98.4 & 3096.1 & 2135.7 & 15.8 & 439.3 & 158.5 & 92.9 & 775.3 & 483.3 & 83.4 \\
\hline 0.04 & 551.7 & 129.8 & 70.8 & 440.3 & 270.4 & 96.7 & 1948.3 & 1304.2 & 11.0 & 354.8 & 140.5 & 84.9 & 522.3 & 307.4 & 83.0 \\
\hline 0.05 & 436.9 & 104.8 & 59.4 & 352.9 & 216.5 & 96.3 & 1298.7 & 813.6 & 8.0 & 275.2 & 109.7 & 78.9 & 266.9 & 139.0 & 81.0 \\
\hline 0.06 & 353.3 & 87.1 & 54.0 & 289.5 & 178.0 & 94.6 & 893.6 & 514.8 & 7.1 & 234.6 & 96.6 & 73.1 & 236.0 & 126.1 & 80.0 \\
\hline 0.07 & 303.4 & 65.2 & 48.7 & 251.7 & 153.3 & 90.9 & 675.7 & 369.9 & 6.7 & 193.7 & 79.7 & 68.5 & 206.4 & 99.6 & 74.8 \\
\hline
\end{tabular}




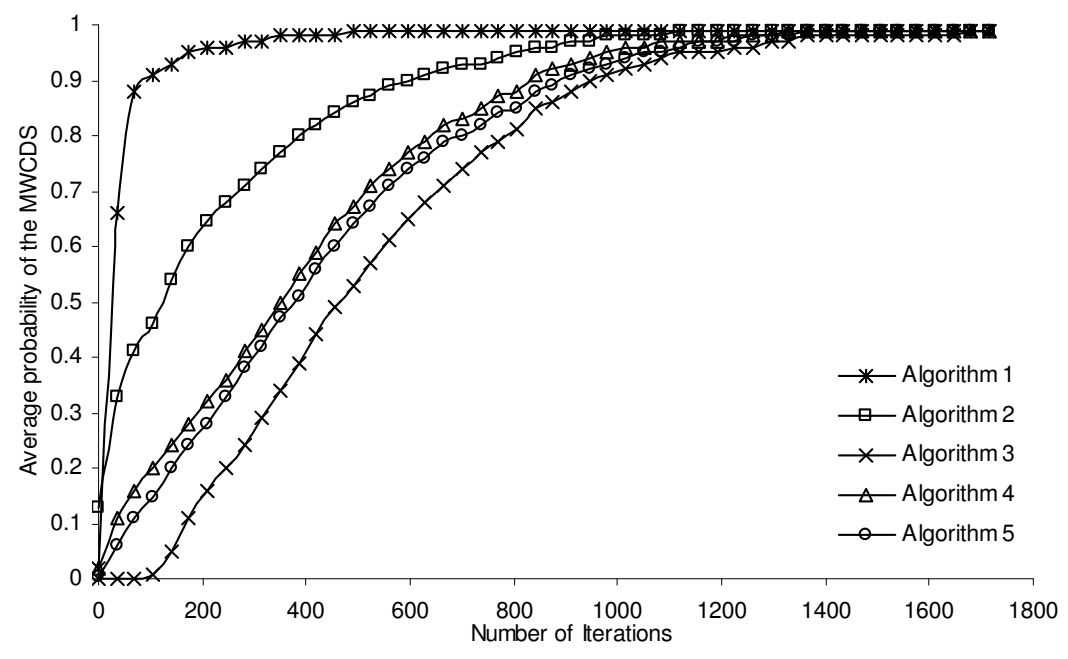

Fig. 12. The probability of the minimum WCDS for Graph 2.

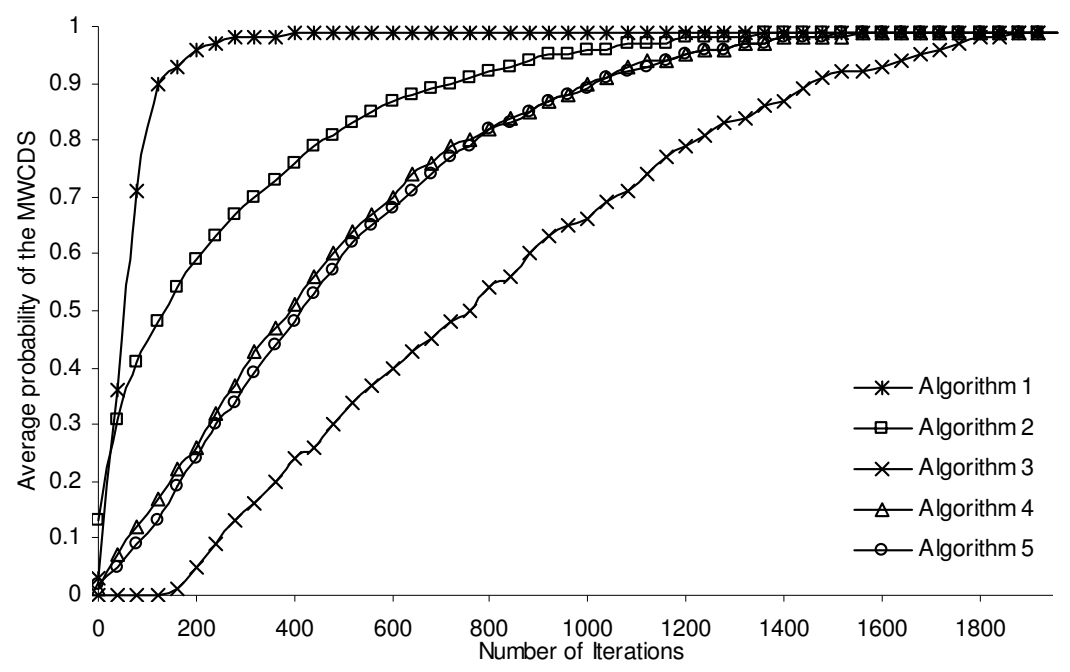

Fig. 13. The probability of the minimum WCDS for Graph 3.

of the minimum WCDS over the converged runs out of 100 runs for both graphs and different algorithms are given in Figs. 11, 12 and 13. Comparing the any time behavior of the proposed algorithms, we observe that algorithm 1 performs best and algorithm 3 performs worst among the algorithms presented in this paper.

To evaluate the scalability of the proposed algorithms, in the second group of the simulations, the proposed algorithms are tested on the dense stochastic graphs. The stochastic graphs used in these experiments are generated based on the method 
Table 7. The sampling rate of the proposed algorithms against the standard sampling method.

\begin{tabular}{|c|c|c|c|c|c|c|c|c|c|c|c|}
\hline \multirow{2}{*}{$\begin{array}{l}\text { Number } \\
\text { of } \\
\text { vertices }\end{array}$} & \multicolumn{2}{|c|}{ Algorithm 1} & \multicolumn{2}{|c|}{ Algorithm 2} & \multicolumn{2}{|c|}{ Algorithm 3} & \multicolumn{2}{|c|}{ Algorithm 4} & \multicolumn{2}{|c|}{ Algorithm 5} & \multirow{2}{*}{$\begin{array}{c}\text { Standard } \\
\text { sampling } \\
\text { method }\end{array}$} \\
\hline & $\mathrm{TS}$ & WCDS & $\mathrm{TS}$ & WCDS & $\mathrm{TS}$ & WCDS & $\mathrm{TS}$ & WCDS & TS & $\overline{\mathrm{WCDS}}$ & \\
\hline 20 & 4796.5 & 1014.0 & 2594.7 & 835.9 & 9216.5 & 5606.0 & 3248.2 & 1763.4 & 6699.2 & 3300.5 & 7663.0 \\
\hline 30 & 5940.0 & 1052.9 & 2916.4 & 850.4 & 7936.5 & 5445.0 & 3080.5 & 1119.0 & 7836.8 & 3456.7 & 13186.0 \\
\hline 40 & 6975.0 & 1002.0 & 3342.2 & 890.5 & 9657.0 & 6513.5 & 4070.5 & 2066.5 & 8304.6 & 3006.2 & 18949.0 \\
\hline 50 & 7874.5 & 1042.2 & 3510.3 & 879.4 & 10671.0 & 6572.4 & 4583.3 & 2690.0 & 8985.4 & 3046.3 & 22134.0 \\
\hline 60 & 8733.2 & 1098.9 & 4034.0 & 868.9 & 11328.4 & 7510.0 & 5259.0 & 2395.0 & 9023.9 & 3721.2 & 26894.0 \\
\hline 70 & 9045.3 & 983.0 & 4084.6 & 894.5 & 15076.2 & 9269.6 & 5445.0 & 2825.0 & 9589.0 & 3960.5 & 30335.0 \\
\hline 80 & 9559.2 & 983.2 & 4216.2 & 865.8 & 15640.9 & 9291.7 & 5670.5 & 3224.5 & 10746.5 & 4172.6 & 33561.0 \\
\hline 90 & 10520.3 & 1213.8 & 4331.1 & 914.6 & 16652.1 & 9625.4 & 5930.4 & 3311.3 & 12184.5 & 4024.9 & 39505.0 \\
\hline 100 & 11428.0 & 1117.1 & 4552.1 & 894.3 & 17186.5 & 10734.7 & 6040.5 & 3519.5 & 12322.0 & 4847.0 & 43686.0 \\
\hline 110 & 11494.1 & 1112.9 & 5078.6 & 921.2 & 19750.0 & 13987.0 & 6570.3 & 3210.2 & 14309.8 & 3598.2 & 49083.0 \\
\hline 120 & 12745.7 & 1215.6 & 4953.0 & 902.6 & 20579.0 & 10028.0 & 6700.1 & 3636.0 & 19593.8 & 7558.8 & 50969.0 \\
\hline 130 & 11876.0 & 1071.7 & 5122.8 & 893.4 & 24869.5 & 13422.5 & 6642.2 & 3515.5 & 19311.5 & 4183.5 & 58787.0 \\
\hline 140 & 13091.4 & 1129.2 & 5222.8 & 910.4 & 27128.0 & 17057.0 & 7010.5 & 3710.0 & 21345.4 & 11618.5 & 64476.0 \\
\hline 150 & 13146.1 & 967.2 & 5276.4 & 900.8 & 31656.0 & 21239.0 & 7230.0 & 3685.3 & 23916.7 & 11012.2 & 66469.0 \\
\hline
\end{tabular}

proposed in Ref. 51 to create the weighted geometric graphs. The weight of each vertex $v_{i}$ (for all $1 \leq i \leq n$ ) is assumed to be an exponential random variable with parameter $\lambda=i / n$, where $n$ is the number of vertices in the graph. It is assumed that the parameters of the underlying probability distribution of the vertex weight are unknown. In such geometric graphs, the vertices are randomly and uniformly distributed in a square area, and an edge connects two given vertices, if they are close enough (if the distance between to vertices is less than $r$ ).

In these experiments, it is assumed that a given number of vertices are randomly and uniformly distributed in a square area of size $100 \times 100$ units, and each two given vertices are connected together if the distance between them is at most 20 . The total number of samples taken from the stochastic graphs by the proposed algorithms is obtained when the number of vertices ranges from 20 to 150 with increment step of 10 and the percentage of the converged runs is 98 percent. All the simulation results given in Table 7 are obtained by running the proposed algorithm on 100 stochastic graphs and averaged over these runs. Table 7 includes the information about the total number of the samples taken from all vertices of the graph (TS) and the total number of the samples taken from the vertices of the minimum WCDS (WCDS) for each of the proposed algorithms as well as the total number of samples taken by the standard sampling method when the confidence level for the WCDS is 98 percent.

The simulation results of the proposed algorithms given in Table 7 show that algorithm 2 performs best and algorithm 3 performs worst among the proposed algorithms. The obtained results also show that the number of samples taken by each of the five proposed algorithms increases as the number of vertices increases. Comparing the total number of samples taken by the proposed algorithms with that of the standard sampling method, we observe that the proposed algorithms 
Table 8. Sampling rate comparison of the algorithm 1 and the standard sampling method for Graph 1.

\begin{tabular}{cccc}
\hline $\begin{array}{c}\text { Convergence rate } \\
(1-\varepsilon)\end{array}$ & $\begin{array}{c}\text { Learning } \\
\text { rate }\end{array}$ & $\begin{array}{c}\text { Sampling rate } \\
\text { (Algorithm 1) }\end{array}$ & $\begin{array}{c}\text { Sampling rate } \\
\text { (Standard sampling method) }\end{array}$ \\
\hline 0.50 & 0.0880 & 255.02 & 3171 \\
0.60 & 0.0690 & 315.79 & 3187 \\
0.70 & 0.0434 & 550.29 & 3118 \\
0.75 & 0.0336 & 715.22 & 3149 \\
0.80 & 0.0281 & 862.60 & 3152 \\
0.85 & 0.0211 & 1154.22 & 3261 \\
0.90 & 0.0122 & 1955.25 & 3404 \\
0.95 & 0.0088 & 2752.33 & 3445 \\
0.99 & 0.0079 & 3049.92 & 3628 \\
\hline
\end{tabular}

significantly outperform the standard sampling method in terms of the sampling rate, especially for the dense graphs.

The remainder of the simulation experiments compares the first proposed algorithm (algorithm 1) with the standard sampling method in terms of the number of samplings. Let $q_{i}$ denotes the initial probability of constructing the minimum WCDS $\omega_{i}$, and $(1-\varepsilon)$ denotes the probability with which algorithm 1 converges to the WCDS $\omega_{i}$ and $\underline{q}(k)$ is updated by algorithm 1 . The learning rate of algorithm 1 needs to be obtained for each error parameter $\varepsilon \in(0,1)$. To do that, parameter $x$ can be obtained from Eq. (20)

$$
\left(1-e^{-q_{i} x}\right) /(1-\varepsilon)=\left(1-e^{-x}\right)
$$

for a given error parameter $\varepsilon$. After some simplification, the equation above is rewritten as an equation of degree $q_{i}$ as follows

$$
\left(e^{-x}\right)^{q_{i}}+e^{-x}(1-\varepsilon)-\varepsilon=0 .
$$

The equation above can be solved by the numerical methods. Learning rate $a$ is then calculated for the given parameter $x$ by solving Eq. (21)

$$
a x / e^{a x}-1=\max _{j \neq i}\left(d_{j} / d_{i}\right) .
$$

The number of samples needs to be taken by algorithm 1 is then determined by running the algorithm for the learning rate obtained from the equation above. The minimum number of samples taken by the standard sampling method, satisfying $\operatorname{prob}\left[\left|\bar{x}_{n}-\mu\right|<\delta\right] \geq 1-\varepsilon$, has also been given in Tables 1,3 , and 5 , where $\delta=0.001$. Comparing the results of algorithm 1 and the standard sampling method, we find that the number of samples taken by algorithm 1 is much less than that of the standard sampling method. These results are given in Table 8.

\section{Conclusion}

In this paper, several learning automata-based algorithms were proposed to solve the minimum WCDS problem in a stochastic graph when the probability distribution function of the weight of the vertices is unknown. To evaluate the proposed 
algorithms, at first, the number of samples needs to be taken from the graph was obtained by the standard sampling method when the approximated value of the vertex weight satisfies a certain confidence constraint. Then, the number of samples taken by the proposed algorithm, where the probability with which the algorithm converges to the WCDS with the minimum expected weight satisfies the given constraint, were calculated and shown to be much less than that of the standard sampling method. As the algorithms proceed, taking advantage of learning automata the process of sampling from the graph is concentrated on the vertices by which the minimum WCDS is constructed. Such an intelligent sampling process meaningfully reduces the number of samples needs to be taken from the graph. For the first proposed algorithm, it was shown that by a proper choice of the parameters of the learning algorithm, the probability with which the given algorithm finds the optimal WCDS is close enough to unity. The simulation results showed the efficiency of the proposed algorithms in terms of the sampling rate.

\section{Appendix A: Standard Sampling Method (SSM)}

Theorem 4. To obtain a confidence level not smaller than $1-\varepsilon$ for the WCDS, it is sufficient to build a confidence with level $1-\varepsilon_{i}$ for every vertex $v_{i}$ such that $\sum_{i=1}^{k} \varepsilon_{i}=\varepsilon$, where $k$ denotes the cardinality of the WCDS.

Proof. The required sample size for each of the vertices to satisfy a confidence level $1-\varepsilon_{i}$ is obtained by using the vertex sampling method described below.

Vertex sampling method Let $\left(x_{1}, x_{2}, \ldots, x_{N}\right)$ be a random sample of random variable $X_{i}$ associated with weight of vertex $v_{i}$ having unknown mean $\mu$ and variance $\sigma^{2}$. If $\bar{x} \pm \sigma / \sqrt{N \varepsilon_{i}}$, where

$$
\bar{x}=\frac{1}{N} \sum_{j=1}^{N} x_{j},
$$

is a $1-\varepsilon_{i} \%$ confidence interval for mean $\mu$, then for each sufficiently small value of $\delta$, there exists a positive number $N_{0}$ such that

$$
p\left\{\left|\bar{x}_{N}-\mu\right| \prec \delta\right\} \succ 1-\varepsilon_{i}
$$

for all $N \geq N_{0}$.

The problem is then to find a confidence region for each of the vertices under which a desired confidence level $1-\varepsilon$ is guaranteed for the WCDS. The confidence region for the WCDS is defined as the intersection $\cap_{i=1}^{k} C_{i}\left(\varepsilon_{i}\right)$ of the confidence regions for the vertices, where $C_{i}\left(\varepsilon_{i}\right)=1-\varepsilon_{i}$ denotes the confidence region for vertex $v_{i}$ and $k$ denotes the cardinality of the WCDS. Using Booles-Bonferroni inequality, ${ }^{52}$ we have

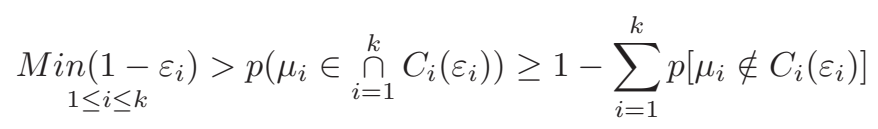


and so

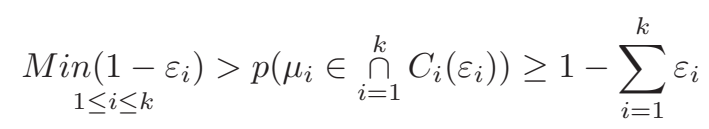

Hence, the confidence level of the WCDS is not smaller than $1-\sum_{i=1}^{k} \varepsilon_{i}$. In this theorem, the objective is to obtain a confidence level not smaller than $1-\varepsilon$ for the WCDS. To achieve this, according to the Bonferroni Correction ${ }^{53}$ it is sufficient to build a confidence with level $1-\varepsilon_{i}$ for each vertex $v_{i}$ such that $\sum_{i=1}^{k} \varepsilon_{i}=\varepsilon$, and hence the proof of the theorem.

\section{References}

1. Y. P. Chen and A. L. Liestman, Maintaining weakly-connected dominating sets for clustering ad hoc networks, Ad Hoc Networks 3 (2005) 629-642.

2. B. Han and W. Jia, Clustering wireless ad hoc networks with weakly connected dominating set, J. Parallel and Distributed Computing 67 (2007) 727-737.

3. R. Rajaraman, Topology control and routing in ad hoc networks: A survey, SIGACT News 33(2) (2002) 60-73.

4. Y. Z. Chen and A. L. Listman, Approximating minimum size weakly connected dominating sets for clustering mobile ad hoc networks, in Proc. Third ACM Int. Symp. Mobile Ad Hoc Networking and Computing (MobiHoc'2002), 2002, pp. 157-164.

5. Y. P. Chen and A. L. Liestman, A zonal algorithm for clustering ad hoc networks, Int. J. Foundations of Computer Science 14(2) (2003) 305-322.

6. K. M. Alzoubi, P. J. Wan and O. Frieder, Maximal independent set, weakly connected dominating set, and induced spanners for mobile ad hoc networks, Int. J. Foundations of Computer Science 14(2) (2003) 287-303.

7. R. G. Gallager, P. A. Humblet and P. M. Spira, A distributed algorithm for minimum weight spanning trees, ACM Trans. Programming Languages and Systems 5 (1983) 66-77.

8. S. Guha and S. Khuller, Approximation algorithms for connected dominating sets, Algorithmica 20(4) (1998) 374-387.

9. O. Dousse, F. Baccelli, and P. Thiran, Impact of interferences on connectivity in ad hoc networks, IEEE/ACM Trans. Networking 13(2) (2005) 425-436.

10. S. Basagni, M. Mastrogiovanni, and C. Petrioli, A performance comparison of protocols for clustering and backbone formation in large scale ad hoc network, in Proc. First IEEE Int. Conf. Mobile Ad Hoc and Sensor Systems (MASS'2004), 2004, pp. 70-79.

11. B. Das and V. Bharghavan, Routing in ad-hoc networks using minimum connected dominating sets, IEEE Int. Conf. on Communications (ICC'97), 1997.

12. I. Chlamtac and S. Kutten, Tree-based broadcasting in multihop radio networks, IEEE Trans. Computing 36(10) (1987) 1209-1223.

13. H. Lim and C. Kim, Flooding in wireless ad hoc networks, J. Computer Communications 24 (2001) 353-363.

14. X. Cheng, M. Ding, D. Hongwei, and X. Jia, Virtual backbone construction in multihop ad hoc wireless networks, J. Wireless Communications and Mobile Computing 6 (2006) 183-190.

15. J. Wu, B. Wu, and I. Stojmenovic, Power-aware broadcasting and activity scheduling in ad hoc wireless networks using CDSs, J. Wireless Communications and Mobile Computing 3 (2003) 425-438. 
16. S. Basagni, D. Bruschi, and I. Chlamtac, A mobility-transparent deterministic broadcast mechanism for ad hoc network, IEEE/ACM Trans. Networking 7(6) (1999) 799 807.

17. R. Ghosh and S. Basagni, Mitigating the impact of node mobility on ad hoc clustering, J. Wireless Communications and Mobile Computing 8 (2008) 295-308.

18. F. D. Tolba, D. Magoni, and P. Lorenz, A stable clustering algorithm for highly mobile ad hoc networks, in Second Int. Conf. Systems and Networks Communications (ICSNC 2007), 2007.

19. Y. X. Wang and F. S. Bao, An entropy-based weighted clustering algorithm and its optimization for ad hoc networks, in Third IEEE Int. Conf. Wireless and Mobile Computing, Networking and Communications (WiMob 2007), 2007.

20. Z. E. Bazzal, M. Kadoch, B. L. Agba, F. Gagnon, and M. Bennani, A flexible weight based clustering algorithm in mobile ad hoc networks, in Proc. Int. Conf. Systems and Networks Communication (ICSNC 2006), 2006, pp. 50-56.

21. T. N. Janakiraman and J. L. Rani, An efficient K-hop weighted domination clustering algorithm for mobile ad hoc networks using ranking, in Int. Conf. Computational Intelligence and Multimedia Applications, 2007, pp. 322-326.

22. W. D. Yang and G. Z. Zhang, A weight-based clustering algorithm for mobile ad hoc network, in Proc. Third Int. Conf. on Wireless and Mobile Communications (ICWMC 2007), 2007.

23. W. Choi and M. Woo, A distributed weighted clustering algorithm for mobile ad hoc networks, in Proc. Advanced Int. Conf. Telecommunications and Int. Conf. Internet and Web Applications and Services (AICT/ICIW 2006), 2006.

24. R. Mellier and J. F. Myoupo, A weighted clustering algorithm for mobile ad hoc networkswith non unique weights, in Int. Conf. Wireless and Mobile Communications (ICWMC 2006), 2006.

25. M. Gerla and J.T.-C. Tsai, Multicluster, mobile, multimedia radio network, J. Wireless Networks 1(3) (1995) 255-265.

26. J. E. Dunbar, J. W. Grossman, J. H. Hattingh, S. T. Hedetniemi, and A. A. McRae, On weakly connected domination in graphs, Discrete Mathematics 167-168 (1997) 261-269.

27. B. Han and W. Jia, Clustering wireless ad hoc networks with weakly connected dominating set, J. Parallel and Distributed Computing 67 (2007) 727-737.

28. I. Chlamtac, M. Conti, and J. Liu, Mobile ad hoc networking: Imperatives and challenges, J. Ad Hoc Networks 1 (2003) 13-64.

29. S. Basagni, M. Conti, S. Giordano and I. Stojmenovic, Mobile Ad Hoc Networking, (IEEE Press, 2004).

30. B. N. Clark, C. J. Colbourn, and D. S. Johnson, Unit disk graphs, Discrete Mathematics 86 (1990) 165-177.

31. M. V. Marathe, H. Breu, H. B. Hunt III, S. S. Ravi, and D. J. Rosenkrantz, Simple heuristics for unit disk graphs, Networks 25 (1995) 59-68.

32. K. S. Narendra and K. S. Thathachar, Learning Automata: An Introduction (PrenticeHall, New York, 1989).

33. M. A. L. Thathachar and P. S. Sastry, A hierarchical system of learning automata that can learn the globally optimal path, Information Science 42 (1997) 743-766.

34. M. A. L. Thathachar and B. R. Harita, Learning automata with changing number of actions, IEEE Trans. Systems, Man, and Cybernetics SMG17 (1987) 1095-1100.

35. M. A. L. Thathachar and V. V. Phansalkar, Convergence of teams and hierarchies of learning automata in connectionist systems, IEEE Trans. Systems, Man and Cybernetics 24 (1995) 1459-1469. 
36. S. Lakshmivarahan and M. A. L. Thathachar, Bounds on the convergence probabilities of learning automata, IEEE Trans. Systems, Man, and Cybernetics, SMC-6 (1976) $756-763$.

37. K. S. Narendra and M. A. L. Thathachar, On the behavior of a learning automaton in a changing environment with application to telephone traffic routing, IEEE Trans. Systems, Man, and Cybernetics SMC-10(5) (1980) 262-269.

38. H. Beigy and M. R. Meybodi, Utilizing distributed learning automata to solve stochastic shortest path problems, Int. J. Uncertainty, Fuzziness and Knowledge-Based Systems 14 (2006) 591-615.

39. J. Akbari Torkestani and M. R. Meybodi, A new vertex coloring algorithm based on variable action-set learning automata, J. Computing and Informatics, 2010, to appear.

40. J. Akbari Torkestani and M. R. Meybodi, Graph coloring problem based on learning automata, in Proc. Int. Conf. Information Management and Engineering (ICIME 2009), Kuala Lumpur, Malaysia, April 3-5, 2009.

41. J. Akbari Torkestani and M. R. Meybodi, Approximating the minimum connected dominating set in stochastic graphs based on learning automata, in Proc. Int. Conf. Information Management and Engineering (ICIME 2009), Kuala Lumpur, Malaysia, April 3-5, 2009.

42. J. Akbari Torkestani and M. R. Meybodi, Solving the minimum spanning tree problem in stochastic graphs using learning automata, in Proc. Int. Conf. Information Management and Engineering (ICIME 2009), Kuala Lumpur, Malaysia, April 3-5, 2009 .

43. J. Akbari Torkestani and M. R. Meybodi, Mobility-based multicast routing algorithm in wireless mobile ad hoc networks: A learning automata approach, J. Computer Communications 33(6) (2010) 721-735.

44. J. Akbari Torkestani and M. R. Meybodi, Weighted Steiner connected dominating set and its application to multicast routing in wireless MANETs, Wireless Personal Communications (Springer Publishing Company, February 2010).

45. J. Akbari Torkestani and M. R. Meybodi, An efficient cluster-based CDMA/TDMA scheme for wireless mobile ad-hoc networks: A learning automata approach, J. Network and Computer Applications, 2010, to appear.

46. J. Akbari Torkestani and M. R. Meybodi, Clustering the wireless ad-hoc networks: A distributed learning automata approach, J. Parallel and Distributed Computing 70(4) (2010) 394-405.

47. J. Akbari Torkestani and M. R. Meybodi, An intelligent global flooding algorithm in wireless ad hoc networks based on distributed learning automata, J. Computer Networks 54(5) (2010) 826-843.

48. S. Lakshmivarahan and K. S. Narendra, Learning algorithms for two person zero-sum stochastic games with incomplete information: A unified approach, SIAM J. Control Optim. 20 (1982) 541-552.

49. K. R. Hutson and D. R. Shier, Minimum spanning trees in networks with varying edge weights, Annals of Operations Research 146 (Springer, 2006) 3-18.

50. S. Holm, A simple sequentially rejective multiple test procedure, Scandinavian J. Statistics 6 (1979) 65-70.

51. Http://dimacs.rutgers.edu/Challenges.

52. F. B. ALT, Bonferroni inequalities and intervals, in Encyclopedia of Statistical Sciences 1 (1982) 294-301.

53. C. E. Bonferroni, Teoria statistica delle classi e calcolo delle probabilità, Pubblicazioni del $R$ Istituto Superiore di Scienze Economiche e Commerciali di Firenze 8 (1936) 362 . 